\title{
Tabularia
}

\section{L'affaire Flavy-Choinet, un exemple de résolution des conflits au lendemain de la reconquête de la Normandie par Charles VII}

The Flavy-Choinet Case, an Example of Conflict Resolution in the Aftermath of the Reconquest of Normandy by Charles VII

Il caso Flavy-Choinet, un esempio di risoluzione dei conflitti all'indomani della riconquista della Normandia da parte di Carlo VII

\section{Lydwine Scordia}

\section{OpenEdition \\ Journals}

Édition électronique

URL : http://journals.openedition.org/tabularia/4646

DOI : $10.4000 /$ tabularia.4646

ISSN : $1630-7364$

Éditeur :

CRAHAM - Centre Michel de Boüard, Presses universitaires de Caen

\section{Référence électronique}

Lydwine Scordia, "L'affaire Flavy-Choinet, un exemple de résolution des conflits au lendemain de la reconquête de la Normandie par Charles VII », Tabularia [En ligne], Richesse du tabellionage normand au Moyen Âge, mis en ligne le 22 juillet 2020, consulté le 24 juillet 2020. URL : http://

journals.openedition.org/tabularia/4646; DOI : https://doi.org/10.4000/tabularia.4646 



\title{
L'affaire Flavy-Choinet, un exemple de résolution des conflits au lendemain de la reconquête de la Normandie par Charles VII
}

\author{
The Flavy-Choinet Case, an Example \\ of Conflict Resolution in the Aftermath \\ of the Reconquest of Normandy by Charles VII \\ Il caso Flavy-Choinet, un esempio di risoluzione \\ dei conflitti all'indomani della riconquista \\ della Normandia da parte di Carlo VII
}

\author{
Lydwine SCORDIA \\ Grhis, Université de Rouen \\ lydwine.scordia@univ-rouen.fr
}

Résumé:

L'acte du 20 mai 1464, passé devant le tabellion de Rouen, reconstitue un conflit d'environ un demi-siècle (entre le temps de la monarchie lancastrienne et l'après-reconquête du duché par Charles VII) entre deux familles, l'une noble (Flavy) et l'autre bourgeoise (Choinet), à propos de la possession et propriété du fief noble de Cliponville (Pays de Caux). L'acte résume les procédures engagées: clameur de haro, procès devant les assises du bailliage (Caudebec), doléance devant l'Échiquier, et finalement compromis infrajudiciaire non contentieux acté devant le tabellion. Il informe sur les pratiques de la Coutume normande au temps de la pacification.

Mots-clés: Coutume normande, clameur de haro, incapacité des femmes, fief noble, infrajudiciaire, monarchie lancastrienne, Pays de Caux, procédures judiciaires, recouvrement de la Normandie (1450), tabellionage

\section{Abstract:}

The act of May 20,1464, passed before the tabellion of Rouen, reconstructs a conflict of about half a century (between the time of the Lancastrian monarchy and the after-reconquest of the duchy by Charles VII) between two families, one noble (Flavy) and the other bourgeois (Choinet), about the possession and property of the noble fief of Cliponville (Pays de Caux). The act summarizes the proceedings initiated: "clameur de haro", trial before the assizes of the bailiff (Caudebec), complaint before the Exchequer, and finally compromise infrajudicial not litigated before the tabellion. It informs on the practices of the Norman Custom at the time of the pacification.

Keywords: Norman Custom, clameur de haro, legal incapacity of women, noble fief, infrajudicial, Lancastrian monarchy, Pays de Caux, judicial proceedings, reconquest of Normandy, notary

\section{Riassunto:}

L'atto del 20 maggio 1464, passato davanti al tabellion di Rouen, ricostituisce un conflitto di circa mezzo secolo (tra il tempo della monarchia Lancasteriana e il post-riconquistamento del 
ducato da parte di Carlo VII) tra due famiglie, una nobile (Flavy) e l'altra borghese (Choinet), a proposito del possesso e proprietà del feudo nobile di Cliponville (Pays de Caux). L'atto riassume le procedure avviate: "clameur de haro", processo dinanzi alle assise del bailliage (Caudebec), reclamo dinanzi allo Scacchiere, e infine compromesso infra-giudiziale non contenzioso messo davanti al tabellione. Informa sulle pratiche della Coutume normanna al tempo della pacificazione.

Parole chiave: Coutume normanna, clameur de haro, incapacità giuridica delle donne, feudo nobile, infra-giudiziale, monarchia Lancasteriana, Pays de Caux, procedure giudiziarie, riconquista della Normandia, notaio

Les chercheurs ayant participé aux récents volumes sur le tabellionage (2011 et 2014) appelaient à ouvrir les lourds registres du tabellionage, très sous-utilisés, pour renouveler les recherches sur les thèmes sociaux, économiques, judiciaires et politiques grâce à cette source rédigée en français par les notaires de la France du Nord, qu'on appelait les tabellions ${ }^{1}$. Mes recherches sur Pierre Choinet, l'auteur du Rosier des guerres, ont été grandement transformées par la découverte de quantité d'actes le concernant dans les registres du tabellionage de Rouen ${ }^{2}$. L'affaire Flavy-Choinet, rapidement évoquée dans le mémoire d'HDR, méritait une étude à part entière.

L'accent est mis dans cet article sur un acte passé devant le tabellionage de Rouen le 20 mai 1464 (Arch. dép. Seine-Maritime, 2 E 1/193), qui reconstitue l'accord de deux familles, l'une noble (Flavy), l'autre bourgeoise (Choinet), en conflit pendant une quarantaine d'années à propos de la possession et propriété d'un fief noble situé en Pays de Caux ${ }^{3}$. L'acte renseigne sur les enjeux économiques et sociaux des deux parties, et sur les procédures de justice mises en branle, dont certaines sont spécifiques au droit normand. Ce qui revient à montrer qu'un simple acte de 56 lignes, extrait d'un des 81 registres rouennais conservés pour la fin du Moyen Âge (1360-1500), apporte de multiples informations sociales, économiques, politiques, judiciaires et infrajudiciaires sur la Haute-Normandie pendant les années 1450-1460, soit lors de la pacification du duché ${ }^{4}$.

Rappelons que le tabellion était un officier royal de la justice gracieuse ${ }^{5}$, qui mettait en forme depuis la fin du XIII e siècle les actes touchant meubles (prêts, apprentissages, transactions commerciales) et immeubles (héritages, mariages,

1. Tabellions et tabellionages de la France médiévale et moderne, 2011; Tabellionages au Moyen Âge en Normandie. Un notariat à découvrir, 2014. Tous mes remerciements à Philippe Cailleux pour avoir relu cet article et m’avoir aidée à résoudre certaines difficultés de lecture de l'acte du 20 mai 1464.

2. SCORDIA, 2019.

3. Voir l'annexe 1: Transcription de l'acte du 20 mai 1464 passé devant le tabellionage de Rouen; cf. fig. 3: Carte des chefs-lieux administratifs aux XIII ${ }^{\mathrm{e}}$ et XIV ${ }^{\mathrm{e}}$ siècles; annexe 4 : Les acteurs de l'acte du 20 mai 1464.

4. Il y a 81 registres conservés dans le tabellionage de Rouen (Arch. dép. Seine-Maritime, 2 E 1/150 à 228) pour la période 1360 ( ${ }^{\text {re }}$ année conservée)-1500, soit presque 92 années, CaIlleux, 2011b, p. 156-157, et son article à paraitre dans les Cahiers Léopold Delisle.

5. Pour la définition des tabellions, leur histoire (de la fin du XIII e siècle à 1687 / 8, date de la création de 12 études notariales) et la description diplomatique des actes, lire ARNoux, 2011 et GuYotJEANNIN, 2011; Roch, 2014a et b. 
immobilier, rentes) ${ }^{6}$. L'enregistrement évolua après 1415 , séparant les actes dans des registres distincts: ceux concernant les biens immeubles (conservés) et ceux des biens meubles (non conservés) 7 .

L'acte du 20 mai 1464, extrait du fonds 2 E 1 des Archives départementales de la Seine-Maritime, correspond à la minute de l'accord passé devant tabellion entre Hector de Flavy et Pierre Choinet, les grosses ayant été envoyées aux parties ${ }^{8}$. Les tabellions devant garder la mémoire des actes passés, leurs clercs ont donc copié des milliers de folios de parchemin $(32 \times 36 / 39 \mathrm{~cm})^{9}$.

Chaque registre commence le plus souvent au jour de Pâques et couvre des durées variables, de six mois à quatre ans selon les périodes ${ }^{10}$. Les tabellions enregistraient tous les jours de la semaine, y compris les dimanches, c'est le cas de notre document, seul acte copié sous la date du «Dimence $\mathrm{XX}^{\mathrm{e}}$ jour de may mil CCCC LXIIII» sous l'autorité du tabellion Guillebert Couppequesne $(1458-1466)^{11}$.

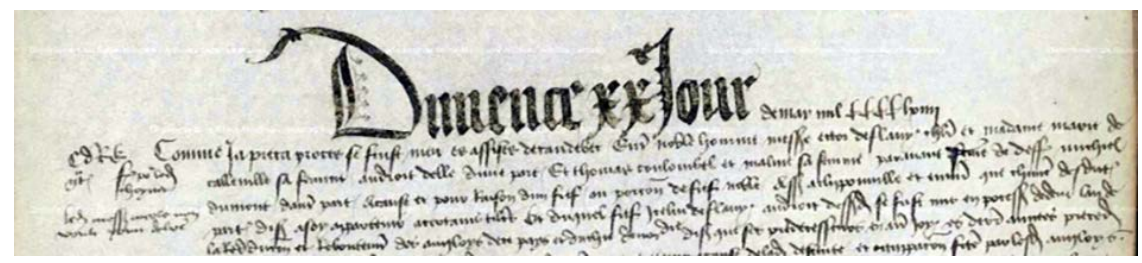

Fig. 1 - Présentation formelle d'un acte de tabellionage:

Arch. dép. Seine-Maritime, 2 E 1/193, dimanche 20 mai 1464, cliché $97^{12}$

6. Les tabellions (le mot s'impose dans la première décennie du XIV ${ }^{\mathrm{e}}$ siècle) avaient d'autres fonctions que celle d'enregistrer les transactions: le règlement infrajudiciaire de l'affaire Flavy-Choinet en est un exemple; ils contribuaient à l'élaboration des cartulaires (CAILlEUX, 2011b, p. 161). Je les ai rencontrés pour l'enregistrement des rôles de l'impôt, voir le cas du fouage de 1446 (BnF, fr. 25910, $\left.\mathrm{n}^{\circ} 590\right)$ levé à Monville (Seine-Maritime, canton de Clères), acté par "Alexandrin Duhamel», commis «soubz Pierre Alatrayme», tabellion de Rouen (1421-1458), SCORDIA, 2019, vol. 1, p. 41-44.

7. Cailleux, 2014, p. 34 .

8. La série $2 \mathrm{E}$ provient du séquestre révolutionnaire. BouCKARD, 1990, p. 300-305. Voir la mise au point sur le fonds dans l'article de Marie Groult et de Justine Ledoux prévu dans le présent dossier de Tabularia: «La numérisation et la mise en ligne des archives notariales: un point d'étape sur les projets des Archives départementales de la Seine-Maritime», Richesses du tabellionage normand. Sur «La chaîne documentaire de la rédaction des transactions dans la juridiction gracieuse », lire Bretthauer, 2019, p. 123-132, en particulier les p. 131-133.

9. Cailleux, 2011b, p. 156.

10. Ibid., p. 157-158.

11. La famille Couppequesne a produit plusieurs tabellions rouennais: Mahiet Couppequesne (1440-1444), celui de l'acte du 20 mai 1464: Guillebert Couppequesne (1458-1466). On retrouve ce dernier nom (mais est-ce le même homme ou son fils?) dans le tabellionage de la sergenterie de Saint-Victor-en-Caux, dépendant du tabellionage rouennais en la vicomté de Rouen, pour les années 1476, 1478; et pour le siège de Clères en 1476. Voir la fort utile liste des tabellions avec les dates d'exercice, réalisée par CAILlEux, 2011b, p. 172-178 et JEAY, 2011.

12. Les registres manuscrits n'étant pas toujours foliotés, j'ai pris le parti de numéroter les clichés des registres numérisés, disponibles sur le site des Arch. dép. Seine-Maritime - l'ensemble du fonds est en ligne depuis septembre 2019. 


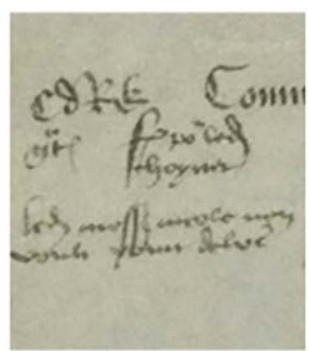

\section{Cd RE}

\section{Gratis fait pour ledit / Choynet}

Ledit messire Nicole n'en / voult point de lettre

Fig. 2 - L'inscription marginale de l'acte du 20 mai 1464

(Arch. dép. Seine-Maritime, 2 E 1/193, cliché 97)

La tarification des actes apparaît dans la marge (pour le scel, pour le registre, pour l'écriture ${ }^{13}$. Dans le cas de l'acte du 20 mai 1464, la marge contient les initiales des tabellions ( $« \mathrm{Cd}$ » pour Cardin Delamare, «RE» pour Raoul Elies) ainsi que deux remarques sur la répartition du coût entre les deux parties (Choinet, Leconte) $)^{14}$ (fig. 2).

Les clercs chargés de recopier les minutes avaient instruction de bien former les lettres pour que cette mémoire notariale soit lisible ${ }^{15}$. Mais, au fil de leurs copies, ils ont multiplié les abréviations juridiques. Après leur résolution dans la transcription in extenso des 56 lignes de l'acte, qu'on trouvera en annexe (annexe 1), il faut encore saisir l'esprit dans lequel les tabellions l'ont rédigé en présence des deux parties après le leur avoir lu. Les noms des deux témoins, indispensables à la validation de l'acte, sont inscrits tout à la fin: «Presens Robinet Delamare et Robinet Le Caron» (ligne 92) ${ }^{16}$.

Le contexte historique de l'accord entre les familles Flavy et Choinet est beaucoup plus large que ne le laisse supposer la date d'enregistrement (20 mai 1464), puisque le tabellion a reconstitué l'historique du conflit qui couvre environ un demi-siècle (avant 1419-1464). On peut le subdiviser en trois périodes:

- la première remonte à l'avant 1419, soit antérieurement à la conquête de la Normandie par les Lancastre, au moment où le fief de Cliponville ${ }^{17}$ appartenait aux Calleville (famille de la femme d'Hector de Flavy);

13. Sur la tarification des actes, lire Labrosse, 1925, p. 234-240. Il existe en fait deux textes de droits coutumiers, CailleuX, 2014, p. 26-28; Bretthauer, 2019, p. 126-131, et p. 147 pour la transcription du «Tarif du tabellionage de Rouen, $\mathrm{XV}^{\mathrm{e}}$ siècle» (Rouen, BM, ms $\mathrm{P}$ 56).

14. Une question et une remarque à propos de ces inscriptions marginales, élucidées grâce à Philippe Cailleux, encore merci à lui. 1) La gratuité pour Choinet est-elle due à son statut de procureur? 2) L'absence de lettre pour Messire Nicole Lecomte, procureur des Flavy, n'est pas exceptionnelle: il n'y a pas toujours de lettre et contrelettre.

15. Sur les modèles stéréotypés des actes, lire Cailleux, 2011b, p. 155-162; Bretthauer, 2014, p. 13-24; BRETTHAUER, 2019, p. 123-152.

16. Ce Robinet (Robert) Delamare est-il parent de Cardin de La Mare, tabellion de Rouen entre 1461-1466 et de Guillaume de La Mare exerçant les mêmes fonctions entre 1475-1482 ? CAILLEUX, 2011b, p. 173.

17. Haute-Normandie, 76 , arrondissement du Havre, commune du nouveau canton de Saint-Valéryen-Caux, située entre Fauville-en-Caux et Yvetot, Dictionnaire topographique de la France, CTHS (en ligne). Voir la carte: fig. 3. 
- la deuxième porte sur les années 1419-1450, c'est-à-dire la période anglaise ${ }^{18}$, pendant laquelle ce sont les Dumont et leurs descendants (Pierre Choinet a épousé leur descendante) qui ont pris possession de Cliponville;

- la troisième traite de l'après « an de la redducion [soumission] et reboutement [expulsion] des Angloys de ce pays et duchié de Normandie» (lignes 6-7), donc après 1450; Hector de Flavy et sa femme sont alors revenus occuper le fief de Cliponville, ce qui a entraîné la réaction des Dumont et les procédures judiciaires et infrajudiciaires jusqu'en 1464 .

Les faits relatés se déroulent dans le duché de Normandie dans le cadre de ses structures administratives et judiciaires royales: bailliage de Caux, assises ${ }^{\star 19}$ de Caudebec, cour de l'Échiquier* à Rouen (lignes 1, 13, 19, 22-24, 33-34, 40, 45-46). Certaines pratiques renvoient à la coutume normande, reconnaissable à ses spécificités patrimoniales, voir la formulation «au droit d'elle $e^{\star}(\text { lignes } 2,6,15)^{20}$, et juridique, voir la clameur de haro* (sommation juridique destinée à réprimer une atteinte à la propriété ${ }^{21}$ (lignes 2, 11). La prévôté* de Paris et le Châtelet sont mentionnés pour attester la procuration de Nicole Lecomte, actant pour les Flavy (lignes 30, 51-54). Cette affaire localement située en Pays de Caux, par conséquent régie par la coutume de Normandie qui est retranscrite dans le Grand Coutumier (milieu du XIII e siècle), s'inscrit dans le contexte de la pacification de la Normandie sous Charles VII et Louis XI.

Les 56 lignes de l'acte qui retrace les termes de l'accord du 20 mai 1464 devant le tabellion de Rouen ne sont pas simples à résumer, car les procédures juridiques sont entremêlées à la généalogie des actants. On peut cependant résumer les étapes principales de l'affaire tout en précisant les définitions nécessaires.

Au temps de l'occupation anglaise du duché de Normandie, Hector de Flavy n'a pu mettre en valeur le fief de Cliponville qui lui venait de sa femme, Marie de Calleville, car le fief a été occupé par 1) Michel Dumont et sa femme Maline [Maleterre] ; 2) puis par la veuve de Michel Dumont, remariée à Thomas Colombel; et 3) par sa fille Jehanne, et ses deux maris, successifs, Robert Le Vigneron et Pierre Choinet. En 1449/1450, la réinstallation de Flavy à Cliponville a provoqué la clameur de haro de Thomas et Maline Colombel pour empêcher cette possession. Le bien et ses revenus sont mis en séquestre* en main de justice. Un premier procès est «meu et pendant* ${ }^{\star}$ devant les assises de Caudebec entre Flavy et Colombel, «recueilli» depuis par Robert Le Vigneron et sa femme Jehanne Dumont (fille de

18. Ces dates (1419-1450) sont données à titre indicatif car la reconquête du Pays de Caux est chaotique (1437-1450); les villes sont prises, perdues, reprises par le parti royal. On en a une preuve avec la double administration anglo-française du bailliage de Caux entre 1437 et 1449, cf. infra.

19. Les mots marqués d'un astérisque sont définis dans l'annexe 2.

20. L'Ancienne Coutume de Normandie, ch. C («De brief de mariage encombré»), p. 243-244. Sur les contextes et les différentes éditions des Coutumiers, lire Neveux, 2011. Les termes judiciaires et infrajudiciaires sont distingués par un astérisque à la première mention, cf. le lexique, dans l'annexe 3; les autres termes sont définis dans le corps du texte entre crochets carrés. Cf. infra.

21. Summa de legibus in curia laicali [1258], Prima pars, quinta distinctio, cap. LIII, p. 141-148. Lire Besnier, 1976, p. 65-67; Cailleux, 2011a, p. 485. Cf. infra et annexe 3. 
Michel Dumont et Maline Maleterre), et enfin par Pierre Choinet, le deuxième mari de Jehanne, l'héritière de Michel Dumont. Hector de Flavy répond au juge-

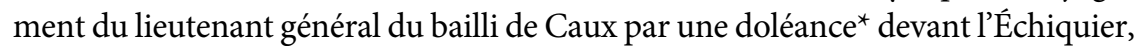
sis à Rouen. Un arrêt de la cour ducale renvoie alors l'affaire devant les assises de Caudebec faute de preuves suffisantes attestant la propriété. Un nouveau procès est engagé entre Nicole Lecomte, le procureur ${ }^{\star}$ des Flavy, et Pierre Choinet, procureur pour sa femme Jehanne Dumont. Mais pour éviter un nouveau renvoi devant le lieutenant général du bailli de Caux, les procureurs des deux parties conviennent d'un accord: Choinet et sa femme auront la possession et propriété du fief avec tous les papiers nécessaires, ils donneront 100 écus d'or aux Flavy. Deux témoins valident l'acte du 20 mai 1464.

Pour mettre en évidence l'historique du conflit et la pacification finale, il convient de commencer par présenter les changements de propriété dans le Pays cauchois sous la monarchie lancastrienne et après «le reboutement des Angloys", puis d'étudier les procédures, y compris la coutume normande et l'infrajudiciaire, qui ont finalement abouti à l'accord du 20 mai 1464 entre les deux parties.

\section{Les changements de "pocession» dans le Pays cauchois sous la monarchie lancastrienne}

La Normandie, que chroniqueurs et actes de la pratique nomment encore le «duchié de Normandie ( (lignes 7), alors qu'elle n'a plus de duc depuis Charles V, connaît de profonds changements entre 1419 et 1450 concernant l'identité du pouvoir politique, ce qui entraîne des répercussions sur la possession de biens immeubles. Les choses changent à nouveau après le recouvrement de Normandie, achevé en 1450. L'affaire Flavy-Choinet s'inscrit dans ce double contexte.

\section{Le duché de Normandie entre France et Angleterre, le fief de Cliponville entre Flavy et Dumont}

Les registres du tabellionage n'ont pas pour mission de chroniquer la période, mais l'histoire transparaît souvent au détour d'une phrase. À partir de 1417, «la venue et descente desdits Angloys» (lignes 8-9) en Normandie et les opérations militaires concertées du roi Henri V et ses frères (Thomas, duc de Clarence; Humphrey, duc de Gloucester) aboutissent à la conquête du duché en deux ans. Après la conquête de la Basse-Normandie à l'été 1418 , le siège de Rouen commence le 29 juillet ${ }^{22}$. La ville capitale, tenue par le capitaine bourguignon, Guy Le Bouteiller, ne reçoit pas de secours extérieurs français et tombe au terme d'un terrible siège de six mois (19 janvier 1419). En plus de la défaite et des morts par famine, les Anglais imposent une composition de 300000 écus d'or qui ruine les Rouennais ${ }^{23}$.

22. Curry, 2006. Voir "Le siège de Rouen vu à travers un registre de tabellionage en 1418 " (Arch. dép. Seine-Maritime, 2 E 1/167), Pigorneau, 1997.

23. Il est précisé que la composition sera payable en écus d'or, valant 22 sous 6 deniers ou en nobles à 45 sous, LARDIN, 1998, p. 101. Sur la ruine due à la composition, lire CAILlEUX, 2011a, p. 98-101. 
Et y moururent par fain plus de XXXm personnes; et si paierent au roy d'Angleterre par composicion IIIc mille escuz d'or, dont pluseurs furent destruitz. Par laquelle rendue, pluseurs autres villes et forteresses se rendirent aux Angloys. Juxta illud in quo continetur anus deffectus panis et pietas occisionis erant in villa Rothomangensis ${ }^{24}$.

La prise de Rouen entraîne celle de la vallée de la Seine, du Pays de Caux, et de tout le duché. Le contexte et les conquêtes amènent Charles VI et Henri V à signer le traité de Troyes (21 mai 1420), qui instaure la double monarchie: Henri V, marié à Catherine, la fille de Charles VI et d'Isabeau de Bavière, devient le gendre du roi fou et l'héritier de France. Commence une période de 31 ans (1419-1450) pendant lesquels la Normandie relève directement de la monarchie lancastrienne, plus encore après le retour de Paris dans le giron français (1436), quand Rouen devient la capitale de la France anglaise ${ }^{25}$.

La guerre en Normandie et le siège de Rouen ont provoqué la mort et la fuite de nombreux seigneurs et simples gens. Des places vides trouent le tissu urbain rouennais et singulièrement les terres du Pays de Caux. L'espace géographique de l'affaire Flavy-Choinet est situé entre Rouen et Dieppe, sur la rive droite de la Seine. Dans un espace faiblement urbanisé, la population s'est rassemblée dans une nébuleuse de villages et hameaux établis sur les coteaux des cours d'eau et les collines ${ }^{26}$. Cliponville se trouve à $60 \mathrm{~km}$ au nord-ouest de Rouen (en rouge sur la carte, fig. 3).

Dès les premières lignes de l'acte du 20 mai 1464, le tabellion résume la position du chevalier Hector de Flavy (v. 1410-1466), qui affirme que «ses predecesseurs» ont possédé le fief de Cliponville jusqu'aux «dernieres annees precedens la venue et descente desdits Angloys», mais qu'ils n'ont pu l'entretenir par la suite «à cause de ladicte descente et occupacion fecte par lesdits Angloys» (lignes 8-10). Les «predecesseurs » dont parle Flavy appartiennent en fait à la famille de sa femme, Marie de Calleville ${ }^{27}$, qu'il a épousée en 1430 . Cliponville est un «fief noble» (ligne 4$)^{28}$.

Les Flavy sont des Picards, d'ancienne extraction noble. Hector, au beau prénom épique, est le fils de Raoul de Flavy et de Blanche de Clermont de Nesle ${ }^{29}$.

24. Pierre Choinet, Le Rosier des guerres, Rouen, BM, ms 996, fol. 57v. Une manchette en latin, soulignée dans le manuscrit, ourle ce passage de la chronique, il s'agit d'un chronogramme. $\mathrm{Si}$ l'on remplace les lettres par des chiffres romains, on trouve la date de 1418: «DeffeCtVs panls et pletas oCCIsIonls erant In VILLa RothoMagensIs », soit M CCCLL VVIIIIIIII.

25. Voir CailleuX, 2003; Sadourny, 2011, p. 165-171; Lardin, 2011, p. 173-184; LeCordier, 2011, p. 205-212.

26. Sion, 1909. Pour une description exemplaire d'une des petites villes du Pays de Caux (géographie, habitat, routes, économie, toponymie, paroisse...), lire la présentation et l'édition donnée par ANGers, 1993, p. 43-45. Sur la typologie des villes et le réseau urbain, lire Neveux, 2006. Cf. fig. 3 : Carte des chefs-lieux administratifs aux XIII et XIV e siècles.

27. La famille des Calleville subit les confiscations du pouvoir anglais: le 28 avril 1419, Henri V donne à Hue Spencer (bailli de Caux entre le 9 mars 1429 et le 29 juin 1430) les domaines confisqués au chevalier Jean de Calleville, HelLot, 1895, p. 112; sur le même sujet, Vautier, 1828, p. 87-88 (don des domaines des Calleville à Spencer).

28. Rien n'est précisé dans l'acte sur les limites du fief de Cliponville, alors que les tabellions sont souvent diserts sur le bornage des terres, objet de nombreux contentieux.

29. Blanche de Clermont de Nesle a d'abord été mariée à Hector de Chartres († 1418), dont est né Regnault de Chartres (vers 1380-1444), archevêque de Reims (1414), chancelier de France (1424). 
Il sert le duc de Bourgogne jusqu'au traité d'Arras de $1435^{30}$. L'acte de tabellionage indique qu'il est «chevalier» (ligne 2) - il a été adoubé en Terre Sainte, ce qui ajoute un grand prestige à son adoubement ${ }^{31}$. Concernant Cliponville, qui lui vient de son mariage avec "Marie de Calleville», la coutume normande (applicable jusqu'à la Révolution, sauf cas particuliers), contenue dans le Grand Coutumier, expose que le patrimoine de chaque époux ne doit pas passer dans la famille du conjoint ${ }^{32}$. L'origine matrimoniale de Cliponville est systématiquement rappelée dans l'acte du 20 mai: Hector de Flavy agit «au droit d'elle» (lignes 2, 6), c'est-à-dire de sa femme. Rappelons que le droit normand frappe la femme d'incapacité juridique durant l'union matrimoniale tout en affirmant l'inaliénabilité dotale: «biens de femmes ne peuvent se perdre», lit-on dans le Grand Coutumier de Normandie ${ }^{33}$ : le régime dotal normand est ouvertement séparatiste. La coutume assure au mari l'usufruit des biens de sa femme et le monopole de la gestion conservatoire des patrimoines. Mais pour les vendre ou modifier leur statut, comme dans le cas de Cliponville, le mari a besoin du consentement de sa femme ${ }^{34}$, d'où la formule présente dans l'acte de 1464: «au droit d'elle ${ }^{35}$. Il en est de même pour l'autre partie: Choinet acte au droit de sa femme, Jehanne Dumont (ligne 15).

Un autre acte du tabellionage rouennais (3o décembre 1454) en lien avec notre affaire permet de préciser la dépendance administrativo-judiciaire de Cliponville: «la terre et seigneurie de Cliponville [est] assise en la viconté de Caudebec »" Cliponville appartient au bailliage de Caux, qui est subdivisé en trois vicomtés royales (Arques, Montvilliers, Caudebec) ${ }^{37}$. Le siège du bailliage finit par être fixé à Caudebec-en-Caux ${ }^{38}$. C'est dans cette petite ville du dernier méandre de la Seine que se tiennent les assises des procès de l'affaire Flavy-Choinet (lignes 1, 34, 46).

Hector de Flavy, né du second mariage de Blanche, est donc le demi-frère de l'archevêque. Il a pour frère aîné, Guillaume de Flavy (v. 1398-1449). L'historiographie s'est partagée sur la responsabilité de Guillaume dans la prise de Jeanne d'Arc à Compiègne (CHAMPION, 1906) et s'est intéressée au meurtre de Guillaume par sa femme Blanche d'Aurebruche (forme du nom flamand Overbreuc), GAUVARD, 2000.

30. Hector de Flavy a possédé L'Armorial Le Breton, le plus ancien armorial figuré conservé en France (950 écus peints ou dessinés), aujourd'hui aux AN sous la double cote: MM684/L et AE/I.25.6. DE Boos et al., 2004.

31. Enguerran de Monstrelet, La chronique d'Enguerran de Monstrelet, en deux livres, avec pièces justificatives, 1400-1444, Louis Douët d'Arcq (éd.), t. 4, p. 436 (à la date de 1431).

32. Musset, 1997; Angers, 2003; Lemonnier-Lesage, 2005; LemonNier-Lesage, 2011 ; LEMONNIER-LeSAGE, 2014. Voir le volume publié à l'occasion de l'exposition «Femmes au travail en Seine-Maritime» (Arch. dép. Seine-Maritime, 13 mars-26 juin 2015): "Tout ce qu'elle saura et pourra faire». Femmes, droits, travail en Normandie du Moyen Âge à la Grande Guerre, 2015.

33. Poirey, 2004, p. 290; Lemonnier-LeSAGe, 2005, p. 157-175 et p. 191-194.

34. TABbagh, 1994, p. 251; Lemonnier-Lesage, 2011; LemonNier-LeSAGE, 2014.

35. Voir note 20. Voir la formulation «Tout ce qu'elle saura et pourra faire» dans l'acte du 26 avril 1395, Arch. dép. Seine-Maritime, 2 E 1/155, fol. 144, cité par RocH, 2015, p. 22.

36. Arch. dép. Seine-Maritime, 2 E 1/186, 30 décembre 1454 (acte de 34 lignes, dont la foliotation, si elle existe, n'est pas lisible car les coins supérieurs des folios sont brûlés).

37. Voir les cartes 1-5 dans l'article de Neveux, 2006, p. 47, 49, 52, 54 et 58 .

38. Hellot, 1895 : présentation historique (p. v-xxxi) et liste des baillis de 1204 à 1790 (p. 1-164). La fonction de lieutenant général du bailli apparaît en 1330, p. xix. 


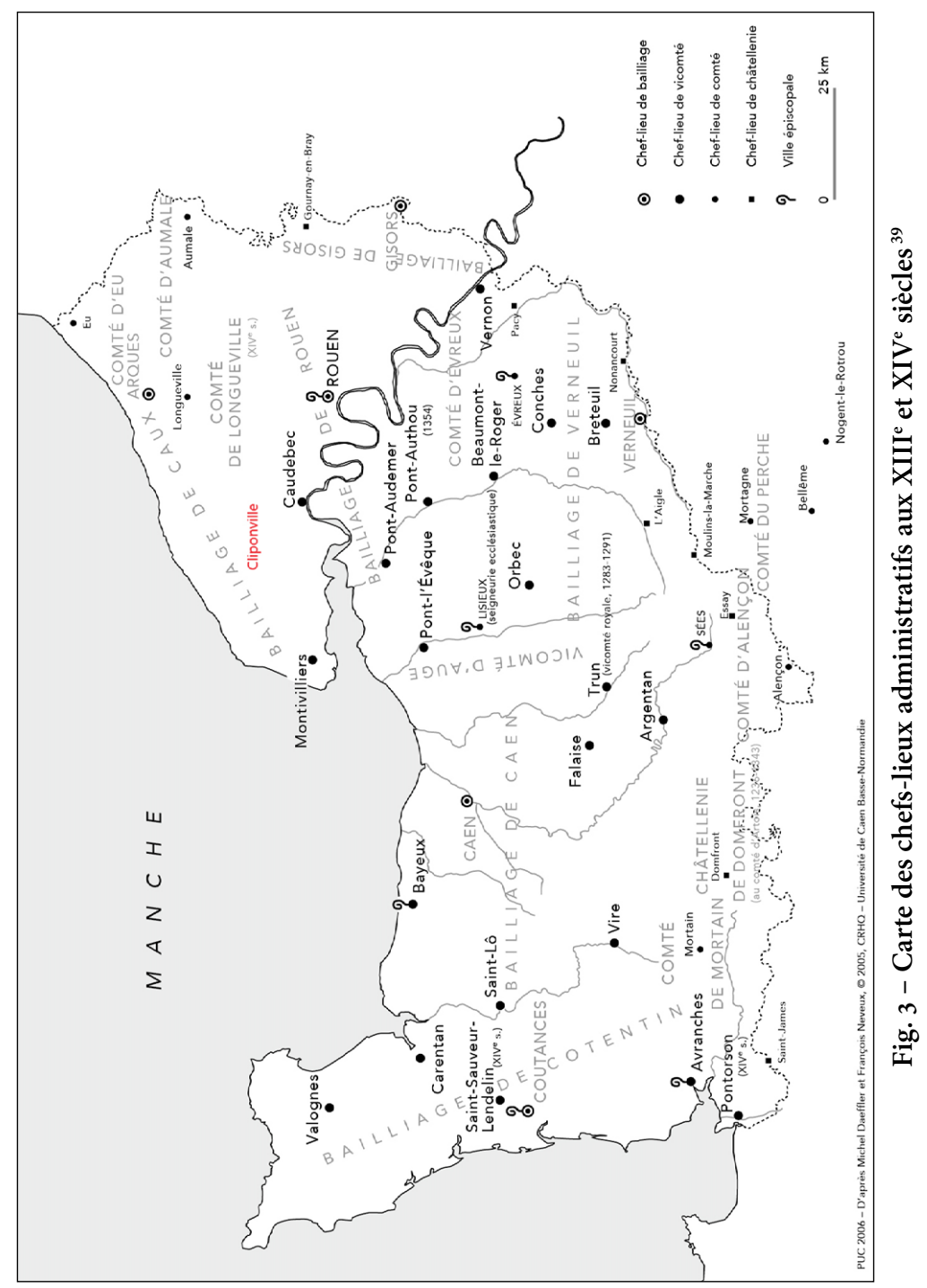

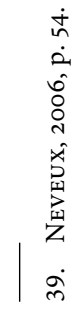


À l'échelle seigneuriale, Cliponville fait partie du fief des Bordes qui, lui-même, dépend de la baronnie de Cleuville. La Normandie comptait environ 4000 fiefs ${ }^{40}$, ce qui ne signifiait pas qu'il y avait 4000 possesseurs car les fiefs étaient souvent divisés ${ }^{41}$. C'est peut-être le cas dans l'affaire Flavy-Choinet, Cliponville est en effet désigné comme «fief ou porcion de fief noble» (ligne 4$)^{42}$.

Très tôt après la conquête de la Normandie, Henri V ordonna de mettre la main sur toutes les places vides et non closes de la ville de Rouen. En cas d'absence de propriétaires, les places furent mises aux enchères. Les nouveaux propriétaires devaient s'engager à les nettoyer et les clore ${ }^{43}$. La situation est similaire à l'échelle du duché, quant au constat de l'absence des propriétaires: la Normandie a perdu nombre de ses seigneurs, tués, enfuis, en tout cas ayant quitté le pays normand ${ }^{44}$. Des biens vont changer de mains sous l'occupation anglaise, soit par confiscation, soit en raison de l'absence (fuite, mort) de leurs propriétaires.

La famille Dumont fait partie des familles qui ont profité de l'absence des seigneurs en Pays de Caux ou/et de la bienveillance anglaise. Il s'agit d'une famille bourgeoise, où les hommes sont gradués en droit et occupent des fonctions juridiques. L'un d'eux, Michel Dumont, se montre particulièrement actif sous le nouveau pouvoir anglais, c'est un avocat, lieutenant du vicomte de Rouen $^{45}$. Il réside à Saint-Godard, la grande paroisse du nord de Rouen ${ }^{46}$, au contact du Pays de Caux où il est déjà possessionné. La monarchie lancastrienne lui offre l'occasion d'augmenter fortement son patrimoine. Le tabellion de l'acte du 20 mai 1464 le cite à plusieurs reprises (lignes 3, 16): Michel Dumont est l'acteur de la possession de Cliponville. À partir d'une date qu'il faut situer entre 1419 et $1432^{47}$, ce bourgeois rouennais occupe et met en valeur «le fief ou porcion de fief noble assis à Clipponville» (ligne 4). Rien n'est précisé dans l'acte de tabellionage sur la nature de cette installation. Est-ce un achat? Il ne semble pas car les descendants de Dumont sont incapables de présenter des documents prouvant leur propriété, même s'ils affirment le posséder «à certains

40. Allmand, 1972, p. 143

41. Angers, 1993, p. 17 (pour la définition du fief qui désigne toute terre d'importance) et p. 74

42. Dans un document à portée fiscale commandé par Louis XII, il est précisé pour la paroisse de Cliponville, sise dans la vicomté de Caudebec, sergenterie de Bans-le-Comte que «En la paroisse de Cliponville y a ung huictiesme de fief nommé le fief de Hardenville, appartenant à Me Guillaume Le Gras, prestres, tenu de la conté de Tancarville. Item. Ung autre huictiesme de fief nommé le fief des Bordes, appartenant à Me Guillaume Le Gras, tenu de la baronnye de Cleuville. Item. Ung huictiesme de fief nommé le fief du Val, appartenant à Pierres de la Mare, tenu nuement du roy", Registre des fiefs et arrière-fiefs du bailliage de Caux en 1503, A. Beaucousin (éd.), Rouen, A. Lestringant, 1891, p. 207. Cf. infra à la note 98.

43. Cailleux, 2011a, distingue différents types de cas, p. 98-133, en particulier aux p. 103-110.

44. On peut lire l'expression «chevalier absent» dans un acte de tabellionage mettant aux prises le «noble homme, messire George, seigneur et baron de Clères, chevalier absent » et Pierre Choinet dans une autre affaire, comparable à celle de Cliponville, Affaire de Clères-Choinet: Arch. dép. Seine-Maritime, 2 E 1/194, lundi 3 juin 1465, cliché 78.

45. BeAurepaire, 1906, p. 284.

46. SCORDIA, 2019, vol. 1, p. 54-56.

47. Car on ne dispose plus d'information sur Michel Dumont après cette date. 
tiltres» (ligne 5$)^{48}$. Est-ce le résultat d'une confiscation anglaise d'un bien confié ensuite à un Rouennais loyal aux Lancastre ou est-ce une occupation d'un bien abandonné ${ }^{49}$ ?

De droit ou de fait, Michel Dumont occupe et met en valeur Cliponville, le fait est attesté par la mention répétée des revenus tirés «dudit fief» (lignes 35-36, 39, 70, 71). Le choix de Michel Dumont de s'installer sur des terres nobles appartenant à une famille éminente, ici les Flavy-Calleville, et dans d'autres actes aux barons de Clères ${ }^{50}$, s'explique justement par le statut noble de leurs terres. Sa propension à choisir des terres nobles relève probablement d'une stratégie d'accès à la noblesse ${ }^{51}$. La possession de fiefs nobles comme le fait de vivre noblement et d'être reconnu comme tel sont des étapes classiques vers l'anoblissement. Michel Dumont et ses successeurs s'y emploient. Il faut aussi considérer que Cliponville se trouve à proximité de biens appartenant déjà aux Dumont en Pays cauchois, autrement dit, la stratégie de Michel Dumont est à la fois sociale (accession à la noblesse) et économique (enrichissement). L'acte de tabellionage reflète une pratique opportuniste d'ascension familiale; il est difficile d'affirmer que les Dumont sont pro-Anglais, disons qu'ils servent un pouvoir qui favorise leurs ambitions ${ }^{52}$.

\section{La succession de Michel Dumont et le recouvrement de la Normandie}

De 1419 à 1449/1450, Michel Dumont et ses descendants exploitent le fief de Cliponville. L'avocat a épousé Maline, dont l'acte du 20 mai 1464 ne donne pas le nom de famille mais que l'on connait par d'autres affaires (Maleterre) ${ }^{53}$. Le couple a eu quatre enfants, dont deux (Colin et Jehanne) sont cités dans notre document (lignes 15-16) ${ }^{54}$.

48. La suite de l'acte montre en réalité qu'il existe des papiers l'attestant. Cf. infra.

49. Cf. supra note 24 .

50. SCORDIA, 2019, vol. 1, p. 67-68 (Affaire Clères-Choinet).

51. Depuis 1275 , l'acquisition de fiefs nobles par un roturier entraînait le paiement d'une taxe (droit de franc-fief) à acquitter en compensation de l'abstention de services dus (ost, conseil...), ORF, t. 1, Toussaint 1275, p. 303-305. Les changements consécutifs à l'occupation lancastrienne de la Normandie poussent Louis XI à commander en 1461 une information sur la noblesse normande. L'enquête avait un arrière-plan financier: soit la noblesse n'était pas prouvée, et le non noble devait acquitter la taille était due; soit elle était attestée, et le nouveau noble devait acquitter une taxe pour faire reconnaître l'anoblissement. L'enquête fut confiée à Rémond de Montfaut, bourgeois de Rouen, directeur général des monnaies de Normandie. Elle eut lieu en BasseNormandie en 1461-1463: Recherche de Montfaut contenant les noms de ceux qu'il trouva nobles et de ceulx qu'il imposa à la taille quoiqu'ils se prétendissent nobles en l'année 1463, P. Labbey de La Roque (éd.), 1818. Cf. infra note 93.

52. Ce qui va à l'encontre de l'historiographie $\mathrm{III}^{\mathrm{e}}$ République (et même $\mathrm{IV}^{\mathrm{e}}$ ) stigmatisant les mauvais Français, collaborateurs des Anglais: Lefèvre-PontALIS, 1893, p. 475-521; 55, 1894, p. 259-305; 56, 1895, p. 433-508; Mollat, 1946.

53. Arch. dép. Seine-Maritime, 2 E 1/186, 30 décembre 1454.

54. Colin, l'aîné, est avocat, il meurt avant le 14 juin 1442, Marion est religieuse, puis il y a Jehanne dont nous allons parler et la plus jeune Éléonore, qui épouse en 1462 Roger Couldren, écuyer. Cf. annexe 4. Beaurepaire, 1906, p. 285. 


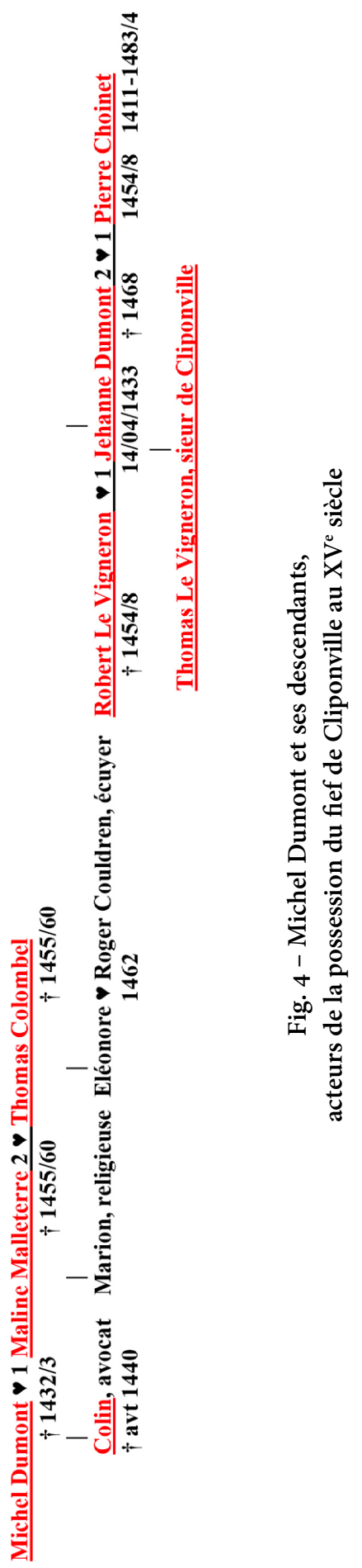

URL: http://journals.openedition.org/tabularia/4646 | DOI : 10.4000/tabularia.4646 
Mais, à la suite du décès de Michel Dumont (après 1432) ${ }^{55}$, déclaré « deffunct» dans l'acte de 1464 (lignes 3, 16), et de son fils Colin, «filz et heritier dudit deffunct Michel Dumont», lui aussi déclaré défunt (lignes 15-16), Cliponville revient à Jehanne, «heritiere» (ligne 15) de son père et de son frère ${ }^{56}$. En 1433, Jehanne se marie une première fois avec Robert/Robin Le Vigneron (contrat du 14 avril 1433 $)^{57}$. Son premier mari, cité aux lignes 16-17, 77, est un bourgeois de Rouen, lui aussi gradué en droit et habitant la même paroisse (Saint-Godard) que les Dumont ${ }^{58}$. Ce fils et petit-fils d'un tabellion de Rouen exerce les fonctions d'avocat et de conseiller en cour laie ${ }^{59}$. Le couple formé par Robert Le Vigneron et Jehanne Dumont constitue le troisième échelon des possesseurs de Cliponville, aussi actif que Michel Dumont à exploiter le fief. Un troisième échelon s'inscrit ensuite dans l'histoire de Cliponville pour des raisons internes aux familles Le Vigneron et Colombel. En effet, le 8 mars 1439 [anc. st.], les deux couples concluent un «accord entr'eulx» qui aboutit à délaisser Cliponville et ses revenus aux Colombel ${ }^{60}$. La possession du fief remonte donc à la génération précédente, celle de la mère de Jehanne et de son beau-père ${ }^{61}$. Un tableau généalogique peut aider à préciser quels ont été les possesseurs du fief (fig. 4, soulignés et indiqués en rouge).

L'histoire aurait pu continuer ainsi, de père (Michel) en fils (Colin), puis en mère (Maline) remariée et enfin en fille (Jehanne), mais le contexte politique et militaire change à partir de 1435 (mort de Bedford; traité d'Arras) et rompt la linéarité des possesseurs Dumont. Le Pays de Caux connaît des soulèvements contre les Anglais (1435-1438), et dès juin 1437, le recouvrement administratif commence avec la nomination d'un bailli de Caux français (Jean Havart) alors qu'il y a toujours un bailli anglais (Thomas Evrygham) ${ }^{62}$. La situation perdure ainsi pendant douze ans, jusqu'au recouvrement militaire de la Normandie par

55. BeAurepaire, 1906, p. 284.

56. Jehanne Dumont est dite «heritere seulle» de son père Michel Dumont, c'est-à-dire unique, Arch. dép. Seine-Maritime, 2 E 1/186, 30 décembre 1454.

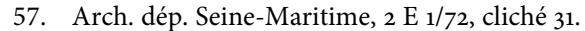

58. Les Le Vigneron sont des bienfaiteurs de la paroisse Saint-Godard de Rouen, Arch. dép. SeineMaritime, G 6609, fol. 27r-28v (1412); Arch. dép. Seine-Maritime, G 6613, cl. 15-20 (1417).

59. Sur le milieu des tabellions rouennais, CAILleux, 2011a, p. 162-164 et p. 173. Henri Le Vigneron est tabellion de Rouen entre 1397 à 1415, Robert Le Vigneron, de 1403 à 1442 - Philippe Cailleux pose la question d'un ou deux tabellions portant le même nom (le père et le fils?) pendant cette période de près de quarante ans. Le fait que le mari de Jehanne Dumont soit avocat et conseiller en court laye n'exclut qu'il ait pu être tabellion, sa formation juridique l'a outillé pour remplir ces différentes fonctions. Voir la reproduction du seing manuel de Robert Le Vigneron, JEAY, 2011, p. $347 \mathrm{n}^{\circ} 52$.

60. Arch. dép. Seine-Maritime, 2 E 1/186, 30 décembre 1454: «Les causes et les lectres sur ce <ont été> faictes et $\mathrm{p}$ <assees> devant Jehan Gouel, tabellion à Rouen [de 1431 à 1438, CAILlEuX, 2011a, p. 173], l'an mil IIIIc XXXIX, le VIIIe jour de mars». Le 8 mars 1439 était un dimanche avant Pâques (le 28 mars cette année-là), faut-il en déduire que les fonctions de Jehan Gouel ont duré jusqu'en 1440 (et non en 1438)?

61. Acte (8 mars 1439) non trouvé dans les registres du tabellionage de Rouen.

62. Hellot, 1895, p. 119-126. 
Charles VII, de ville en ville: Rouen (octobre 1449); Cherbourg (12 août 1450) ${ }^{63}$. Charles VII devient «Le trés victorieux roy de France», comme l'indique l'inscription du célèbre portrait de Fouquet. La double monarchie, réunie sous l'autorité d'Henri VI, s'effondre même si l'Angleterre conserve encore la Guyenne et Calais.

De 1450 à 1453, Charles VII ordonne la pacification de la Normandie et mène la guerre en Aquitaine. Le roi avait déjà évoqué en 1429 la question des changements de propriété alors que le royaume était loin d'être reconquis. Dans l'édit de Compiègne du 22 août 1429, Charles VII avait déclaré que tous ses fidèles sujets seraient remis en possession de leurs biens sans qu'ils aient à entrer en compte avec leurs détenteurs (anglais ou français pro-anglais) ${ }^{64}$. L'édit n'ayant pu être enregistré en raison de la guerre, Charles VII le confirme en octobre 1450 dans un contexte plus réaliste puisque ce qui n'était qu'une profession de foi est devenu un acte royal d'actualité. Si la paix entre la France et l'Angleterre n'est pas encore à l'ordre du jour, le temps est venu de penser à la pacification du royaume ${ }^{65}$.

Le retour à la situation ante bellum s'avère difficile. La reconquête provoque le retour des anciens propriétaires en Normandie. Vraisemblablement dès 1450/1451, Hector de Flavy et sa femme Marie de Calleville reviennent à Cliponville:

«Icelui de Flavy, au droit dessusdit, se fust mis en pocession dedens l'an de la redducion et reboutement des Angloys de ce pays et duchié de Normandie» (lignes 6-7).

Contrairement à l'édit de Charles VII, anciens propriétaires et nouveaux possesseurs vont s'opposer et «entrer en compte». Le recouvrement du duché met en évidence deux réalités nettement distinguées par l'acte de tabellionage de 1464: la "pocession» (lignes 6, 11) et la propriété (lignes 34-35) du fief de Cliponville. Les descendants de Dumont et le couple Flavy, prétendent se maintenir en saisine du fief; dès lors, «la charge de la preuve incombe aux deux parties ${ }^{66}$.

63. Charles VII invita à inscrire le 12 août dans les calendriers pour rendre grâce à Dieu de la victoire. Voir deux témoins manuscrits du Rosier des guerres de Pierre Choinet (Londres, Br. Library, Harley, MS 4406, fol. 4v). Sur le 12 août, «la feste du roy», lire BeAune, 1985, p. 182-187; Contamine, 2012, p. 342-348.

64. L'édit de Compiègne est confirmé le 28 octobre 1450 (ORF, t. 14, p. 102-105). Une addition concerne la Normandie car, dit le roi, plusieurs vont au contraire de l'édit royal dans le duché (p. 105-106). Lire du Fresne de Beaucourt, t. 2, p. 613. Précisons que 1450 est une année jubilaire, selon le livre du Lévitique ( $\operatorname{Lv} 25,8-55)$, «tous heritaiges et possessions paravant vendues, usurpees ou aultrement alienees oudit retournoient à leur propre seigneur, comme il appert ou XXVe et XXVIe chapitre du livre de Levitique», Gilles LE Bouvier, dit le Héraut Berry, Les chroniques du roi Charles VII, p. 353 (ajout en marge d'un des manuscrits des Chroniques, Troyes, BM, ms 790). Les contextes vétéro-testamentaire, pontifical (Nicolas V) et royal (Charles VII) convergeaient.

65. Contamine, 1993.

66. BESNIER, 1976, p. 66 (pour la citation entre guillemets). 
Cette première partie atteste déjà la fécondité thématique de l'acte du 20 mai 1464. Le document apporte des informations sur les événements militaires (conquête anglaise du duché) qui transforment la situation de deux familles, l'une d'ancienne noblesse (Flavy), l'autre bourgeoise évoluant vers la noblesse grâce à la possession de terres nobles (Dumont). La gestion du fief de Cliponville ajoute encore les thèmes économique et financier aux approches politiques et sociales. L'ensemble est à appréhender à des échelles géographiques locale et régionale. Il reste maintenant à étudier les différentes procédures de justice qui ont couru pendant 14 ans, de 1450 à 1464, avant d'arriver à la tractation du 20 mai.

\section{Les registres du tabellionage de Rouen : la mémoire des procédures}

Au lendemain de la reconquête de la Normandie par Charles VII, plusieurs procès mettent aux prises les descendants de Michel Dumont avec les anciens propriétaires de biens nobles occupés pendant la période lancastrienne: «chacune desdictes parties disent à soy appartenir à certains tiltres» (ligne 5). Hector de Flavy l'affirme: le fief de Cliponville appartenait à la famille de Marie de Calleville, qu'il a épousée en 1430, donc sous la double monarchie, quand ce chevalier servait le duc Philippe le Bon de Bourgogne. Les descendants de Michel Dumont affirment aussi que le fief leur appartient. L'édit de Compiègne et les procédures normandes forment l'arrière-plan de cette deuxième partie consacrée aux différentes actions juridiques avant l'accord non contentieux final. Les deux familles en présence manifestent autant leur âpreté que leur pragmatisme et leur capacité à transiger.

\section{Historique des différentes procédures devant la justice}

Avant d'en arriver aux termes de l'accord, les tabellions rappellent le contexte et les circonstances de chaque procédure, ce qui permet de reconstituer l'histoire judiciaire de l'affaire de Cliponville dans le bailliage de Caux (assises) et devant la cour du duché (Échiquier).

L'acte de 1464 fait état de deux manières d'engager le procès: la première est accusatoire ("clameur de haro»), elle mène à la seconde qui est inquisitoire (la «veue $\left.{ }^{\star}\right)$. À peine Flavy s'est-il «mis en pocession» du fief, que Thomas Colombel et Maline Malleterre, les occupants de Cliponville depuis l'accord du 8 mars 1439, prononcent une «clameur de haro" contre le chevalier (ligne 11). Le couple s'estime menacé dans sa possession d'un bien et crie «haro", selon le droit normand. Il s'agit d'une "sommation juridique de surseoir à toute entreprise et de se rendre immédiatement devant la justice pour faire statuer sur le différend ${ }^{67}$. La clameur est prononcée par Guillebert Couppequesne (lignes 11-12) à la demande du couple Colombel. Notons que ce haro civil est prononcé par les possesseurs

67. Arch. dép. Seine-Maritime, 2 E 1/186, lundi 30 décembre 1454. BESNIER, 1976, p. 65 (pour la citation entre guillemets). 
de Cliponville, et non par le couple Flavy qui se dit propriétaire du fief. Cette procédure accusatoire empêche la possession d'Hector de Flavy et place le bien de Cliponville et ses revenus sous séquestre (lignes 35-36). Elle va entraîner le paiement d'une amende* dite «dudit haro» (lignes 44-45), dont il faudra préciser qui aura à la payer.

Chacune des parties prétend se maintenir: l'une (Colombel) accuse Flavy d'une entreprise indue, l'autre (Flavy) accuse Colombel d'un cri de haro injustifié ${ }^{68}$. La clameur de haro amorce un débat possessoire qui relève de la compétence des plaids d'héritage ${ }^{69}$. L'action possessoire (ligne 29) tend à maintenir ou rétablir la possession considérée comme un état de fait, distinct de la propriété, pour mettre fin à un trouble ${ }^{70}$. L'action pétitoire est une revendication juridique tendant à obtenir la restitution d'un bien en propriété. L'enjeu du conflit Flavy-Colombel est la possession de Cliponville, mais à terme il s'agit de déterminer le «droit principal et proprietaire dudit fief» (ligne 34-35).

La distinction faite entre la possession et la propriété permet au perdant sur le plan de la possession (ici Hector de Flavy) de reprendre l'action sous l'angle de la propriété, ce que va faire le chevalier ${ }^{71}$. Au terme d'une longue procédure, c'est finalement Flavy qui renonce à «pretendre, demander et reclamer, tant en petitore $^{\star}$ que pocessore $^{\star}$ à quelque droit, tiltre, raison ou moyen ce soit en icelui fief» (ligne 68-69).

«Ja pieça», indique l'acte, un premier procès a eu lieu au civil aux assises de Caudebec, donc à l'échelle du bailliage, devant le lieutenant général du bailli (lignes 1, 21-22), entre Flavy, au droit de sa femme Marie de Calleville, et Colombel, au droit de sa femme, Maline Malleterre (lignes 2-3). Une instruction est faite sur place avec enquête, on l'appelle «la veue» en droit normand (ligne 18). La date du procès n'est pas autrement datée que par «ja pieça» (ligne 1), mais on peut avancer qu'il s'est tenu entre 1449/50 et 1454, soit entre le «reboutement des Angloys» (et la clameur de haro prononcée par les Colombel) et un acte passé devant le tabellionage de Rouen (30 décembre 1454) qui nous informe que Cliponville a encore changé de main et que le fief est passé à Jehanne Dumont et son mari Robin Le Vigneron.

Procédures judiciaires et histoires de famille s'entrecroisent et complexifient l'affaire de Cliponville. En effet, dans l'acte de 1454, le tabellion précise les termes de l'accord entre:

- d'un côté «Thomas Colombel et Maline, sa femme, en precedent femme de deffunct Michel Dumont»,

68. BESNIER, 1976, p. 66.

69. Cailleux, 2011a, p. 485 (glossaire).

70. Glasson, 1890; Besnier, 1976, p. 65; Poumarède, 1976, p. 597-598. "Les premiers procès possessoires de 1292 et 1293 montrent bien que la contestation porte sur la saisine», Arresta communi Scaccarii, Ernest Perrot (éd.), 1910, nº 8o, cité par Besnier, 1976, p. 65 et note 8, p. 65. Voir le Lexique judiciaire et infrajudicaire de l'acte du 20 mai 1464, annexe 2.

71. Polrey, 2004, p. 305. 
- et de l'autre «Robin Le Vigneron et Jehannecte, sa femme, fille dudit feu Michel Dumont et de ladicte Maline, icelle Jehannecte, heritere seulle dudit feu Dumont $»^{72}$

Alors que Jehanne Dumont et Robert Le Vigneron avaient cédé Cliponville à Maline Malleterre et Thomas Colombel en 1440 (8 mars 1439, anc. st.), l'acte de 1454 précise que Thomas et Maline Colombel renoncent à «ladicte terre de Cliponville ${ }^{73}$ car elle «est en procés à l'encontre de messire Ector de Flavy et [quelques mots manquent, le coin du folio étant brûlé] à clameur de haro et sequestree en main de justice». Les Colombel, beau-père et mère de Jehanne, ont d'autres biens, comme «la terre et seigneurie de Neufville soubz le Vieil Rouen, pieça acquise par ledit feu Michel Dumont de deffunct, hault et puissant seigneur, monseigneur George, baron de Clere, chevalier », qui «de present comme inutille et de petite valleur, tant pour cause de guerres qui ont couru, qu'elle est loing de ceste ville de Rouen, que aussi que elle couste grans deniers à relever des mains des seigneurs dont elle est tenue avant que on en puisse avoir le joissement». Le couple Colombel se plaint d'avoir à faire "grans frais à grant a long trait de temps», ce qu'ils ne peuvent plus assumer «attendu leur estat, fiebesse et entiquité». C'est pourquoi la mère (Maline) et la fille (Jehanne) s'entendent en 1454 lors d'un nouvel accord passé devant le tabellion, qui annule celui de $1439^{74}$ :

Colombel et sa femme déclarent «avoir quicté, cedé, transporté et du tout en tout delaissé affin d'eritage ausdits Vigneron et sa femme et aux hoirs et ligné du costé de ladicte Jehannecte tout et tel droit, action, posté, seigneurie et reclamacion que ilz ont et pevent avoir et reclamer esdictes terre de Cliponville, vingt livres de rente d'Aulage, et en ladicte terre de Neufville, et s'en dessaisirent et en saisirent ledit Vigneron et sa dicte femme».

Jehanne Dumont et son premier mari, Robert Le Vigneron, récupèrent donc le fief (et le procès appendant avec les Flavy) moyennant le paiement de 35 livres de rente par an le temps de «la vie d'icelle [Maline] durant seullement ${ }^{75}$. Entre 1454 et 1458 , Robert Le Vigneron meurt, Jehanne Dumont, sa veuve, se remarie avec "honnorable maistre Pierre Choynet, docteur en medicine» (lignes 17-18), le futur médecin et astrologue de Louis XI et l'auteur du Rosier des guerres et du Livre des trois âges ${ }^{76}$. Pierre Choinet a alors entre 43 et 47 ans $^{77}$, c'est un bourgeois né à Monville et résidant à Rouen dans la paroisse Saint-Godard où il assure la fonction de trésorier de la fabrique en $1454^{78}$. Une

72. Arch. dép. Seine-Maritime, 2 E 1/186, lundi 30 décembre 1454

73. Le clerc a écrit «Camponville», il faut lire «Cliponville», ibid.

74. Ibid.

75. Ibid.

76. Pierre Choinet, Le Livre des trois âges, Lydwine Scordia (éd.), 2009; ID., Le Rosier des guerres, Lydwine Scordia (éd.), à paraître à la SHF. SCORDIA, 2019, vol. 1.

77. L'âge au mariage de Choinet est tardif. Est-ce son premier mariage?

78. Beaurepaire, 1906, p. 284. 
commune inscription sociale et géographique a rapproché les deux époux. Fort avisé, le docteur en médecine, Pierre Choinet, épouse une riche veuve, dont il administre les biens tout en poursuivant les procédures en cours. Les registres $\mathrm{du}$ tabellionage rouennais contiennent de nombreuses pièces où Choinet acte au droit de sa femme, "au droit d'elle». L'acte de 1464 précise de surcroît que Choinet assure «en personne» la fonction de "procureur de sadicte femme» (ligne 31). Il le fait en raison de l'incapacité juridique de sa femme et de la responsabilité qui lui incombe, en tant que mari, de conserver l'inaliénabilité des biens de Jehanne Dumont ${ }^{79}$. Les biens appartiennent à Jehanne et, après elle, au fils prénommé Thomas / Thomassin qu'elle a eu de Robert Le Vigneron. Pierre Choinet œuvre à maintenir Cliponville dans le patrimoine de Jehanne, il est l'artisan de la poursuite de l'enrichissement des Dumont-Le Vigneron.

On peut résumer la liste de ceux qui ont eu le possessoire de Cliponville et qui ont recueilli les procédures en cours:

- Michel Dumont

- Colin Dumont, son fils aîné

- Jehanne Dumont, sa fille, et son premier mari Robert Le Vigneron

- Maline Malleterre, la veuve de Michel Dumont, remariée à Thomas Colombel,

- à nouveau Jehanne Dumont et Robert Le Vigneron, puis Jehanne Dumont avec son second mari Pierre Choinet.

Du côté de l'ancien propriétaire, Hector de Flavy et sa femme, la situation est plus stable, le couple est toujours en vie pendant la durée du conflit, mais le chevalier a choisi un procureur, appelé «messire Nicole Lecomte» (lignes 30, 47, $51,82)^{80}$, qui désormais suit l'affaire en justice pour le couple.

Après la «clameur de haro", le procès entre Flavy et Colombel, tenu "pieça» (1450/4) devant les assises de Caudebec selon la procédure inquisitoire («la veue $)^{81}$, n’aboutit pas. Les Le Vigneron et Choinet recueillent le procès. Une nouvelle "veue» est "tenue et plaidee es assises», parfaitement valide, lit-on, car «la veue [a été] passee sans saon ${ }^{*}$ », c'est-à-dire sans récusation des témoins, «en propoz, responce, repplique ${ }^{\star}$ et dupplique ${ }^{\star}$ (lignes 18-20). Les termes juridiques sont précis sous la plume du tabellion. Le procès aboutit à

79. Pierre Choinet est gradué en droit, comme on l'apprend dans l'acte du 12 novembre 1493 exposant le remariage de Marion de Remy, seconde femme de Pierre Choinet, avec Angelot Morel: feu Pierre Choinet «en son vivant licencié en loix et docteur en medecine», Arch. dép. Seine-Maritime, 2 E 1/218, mardi 12 novembre 1493, clichés 138-139.

80. Cailleux, 2011 b a relevé le nom de Colin Le Conte, tabellion dans la sergenterie de Saint-Victoren-Caux 1457, 1459, 1462, avec Philippe Lestourny en 1463, 1469, p. 178. Est-ce le même homme que «messire Nicole Lecomte» de l'acte de 1464 ? On trouve une famille Le Conte dans l'armorial des familles nobles de Normandie, ce qui pourrait justifier l'avant-nom «messire», inscrit par les tabellions pour qualifier le procureur des Flavy.

81. Poirey, 2004, p. 307. 
un jugement «fait par Robinet Helart, lieutenant general du bailli de Caux» (lignes 21-22), qu'on peut dater entre 1459 et $1461^{82}$.

Le jugement de Robert Hellart a dû être favorable aux descendants des Dumont, les nouveaux possesseurs - on aurait pu penser qu'il serait favorable aux anciens propriétaires, mais ce n'est pas le cas, il atteste la validité de la clameur de haro -; aussi Hector de Flavy en appelle-t-il à la juridiction supérieure et pose une «dolleance» devant l'Échiquier «à present seant à Rouen» (ligne 23).

La cour de l'Échiquier constate alors que «ladicte matiere n'eut peu bonnement estre congneu " (lignes 24-25), elle annule la doléance et prononce un arrêt qui renvoie l'affaire aux termes du jugement fait par Robinet Helart «esdictes assises de Caudebec ». Une nouvelle "veue», apparemment difficile (lignes 40-44) est lancée: les deux parties reprennent alors «leur matere en propoz, response et repllique sur le pocessoire dudit fief» (lignes 28-29) pour savoir «sommierement et de plain* auquel d'eulx ledit fief appartient en principal» (lignes 32-33). L'acte continue de clairement distinguer possession et propriété.

L'immobilisme de l'affaire de Cliponville n'est qu'apparent. Les positions des deux parties évoluent après l'arrêt de l'Échiquier, non que Flavy ou Choinet renonce à Cliponville, mais leurs tractations intègrent désormais un paramètre financier ${ }^{83}$. Depuis le séquestre consécutif au haro, le fief a rapporté des revenus, également mis en main de justice. Les plaideurs s'en inquiètent et se mettent d'accord par un appointement qui va servir de base au nouveau procès: les «fruictz et revenus» placés «en sequestre» depuis le haro seront partagés par moitié, de même pour les revenus "pendant le procés» à venir; une autre clause précise que celui qui aura perdu restituera l'argent à celui qui aura gagné (lignes 38-40). L'arrêt prévoit également que si le procès n'aboutit pas «à la prouchaine assise» (lignes 45-46), l'amende du haro sera payée «par main commune», c'est-à-dire par moitié (lignes 44-45).

L'approche financière a visiblement fait progresser le conflit Flavy-Choinet, et c'est sur cette nouvelle base que l'affaire est « renvoyee à la prouchaine assise dudit lieu de Caudebec, jouxte l'arrest dudit Eschiquier» (lignes 45-46). L'affaire dure déjà depuis 14 ans devant les cours de justice, mais en fait depuis 45 ans depuis qu'il y a eu dissociation de la possession et de la propriété. Si le fief rapporte des revenus mis en séquestre, le procès occasionne des frais de procédure qui s'accumulent sans que l'acte en précise le montant, il faut aussi mentionner la préoccupation mentale et psychologique subie dans un pareil conflit. Précisons encore que le couple Pierre Choinet et Jehanne Dumont doit régler d'autres affaires du même type en Pays de Caux ${ }^{84}$.

82. Robert Hellart apparaît en tant que lieutenant général du bailli Guillaume Prunelay, dont les dates d'exercice sont 26 mai 1459-juin 1461. Hellot, 1895, p. 129-13o. Robert Hellart reste lieutenant général du bailli de Caux sous Jacques Rouault (1461-1465), p. 131-132; Antoine d'Aubusson (1466-1477), p. 132-135; Pierre Blosset (1478-1498), p. 136-139. Son exercice court pendant près de 40 ans $(1459-1494)$ - mais est-ce le même ou son fils?

83. L'argent s'avère souvent indispensable pour apporter de la souplesse à la résolution des conflits, à la fois comme moyen et comme fin. GAUVARD, 2001, p. 384-385.

84. SCORDIA, 2019, vol. 1, p. 63-73 
La résolution $d u$ "descord" à l'initiative des procureurs Nicole Lecomte et Pierre Choinet

L'affaire de Cliponville est à appréhender ligne après ligne en croisant les paramètres familiaux, judiciaires et infrajudiciaires. En 1464, les deux parties ont épuisé les différentes procédures, certaines sont spécifiques à la Normandie (clameur de haro, Échiquier), d'autres relèvent de la justice ordinaire (assises). À la veille d'un renvoi devant les assises de Caudebec en vertu de l'arrêt de l'Échiquier, qui promet d'aboutir aux mêmes conclusions ${ }^{85}$, des "conseulx" (ligne 49) interviennent pour amener le procureur Nicole Lecomte pour Hector de Flavy et «ledit Choynnet» pour lui ${ }^{86}$ et sa femme à s'accorder sur un appointement "pour eviter à icelui procés et pour paix et amour nourrir entr'eulx» (ligne 48) et "pour fuyr à la longueur d'icelui procés» (ligne 79) ${ }^{87}$.

L'infrajudiciaire entre de plain-pied dans l'acte de tabellionage, il est repérable par la modification de l'argumentation: l'âpreté et la détermination des deux familles aux prises depuis 1450 cèdent le pas à des considérations plus générales (paix, amour) et d'autres très concrètes (longueur du procès, autrement dit son coût et les soucis qu'il entraîne). Des tractations avaient dû commencer en marge des procédures, elles sont menées par les "conseulx" dont l'identité n'est pas précisée dans l'acte; ce sont vraisemblablement des prud'hommes, des arbitres, des médiateurs de paix, qui interviennent en vue de résoudre les conflits ${ }^{88}$. Ils sont peut-être intervenus dès 1449/1450, mais ce n'est qu'en 1464 que leurs avis sont entendus, le temps a fait son œuvre. Il n'y a pas d'antagonisme entre justice publique et pratique de l'arbitrage amiable $^{89}$, les deux se superposent et se complètent pour parvenir à rétablir la paix. La radicalité des positions des Flavy et Choinet («chacune desdictes parties disent à soy appartenir à certains tiltres ») a laissé la place à la recherche d'un compromis ${ }^{90}$.

La sentence arbitrale du 20 mai 1464 est passée devant le tabellion de Rouen (Guillebert Couppequesne), soit en dehors du tribunal des assises et de la cour de l'Échiquier, ce qui n'est pas rare ${ }^{91}$. Pour ce faire, Nicole Lecomte, le procureur «dudit Ector de Flavy», a dû obtenir une autre "procuracion» (25 avril 1464),

85. Sur les difficultés de la «veue» aux lignes 40-44: l'appointement est trouvé «ou cas que à la prouchaine assise, aprez ladicte veue fecte, la matere n'eust prinse fin et qu'il y eust aucun delay prins par lesdictes parties plaidant leur matere en l'absence des gens d'icelle veue, comme fere eussent peu en leur presence, parce que se ilz se feussent appointés en preuve, leurs fais eussent esté escrips, et celui qui l'eust eue à fere l'eust fecte, ainsi qu'il eust appartenu ».

86. Pierre Choinet a participé à la mise en valeur de Cliponville.

87. Sur l'importance politique et judiciaire de l'amour, SCORDIA, 2012, p. 129-143.

88. L'arbitrage, 2009.

89. Gauvard, 1991, t. 1, p. 19-20. Lire CARrier, 2001.

90. La situation inverse peut se produire quand l'arbitrage échoue et que la justice prend le relais et aboutit à un jugement.

91. Billoré, Mathieu et Avignon, 2012, ch. 5 «Résoudre les conflits, rétablir la concorde», p. 147162; CARRIER, 2001, p. 253. Sur l'importance de l'institution notariale en matière de règlement des conflits: MANEUVRIER, 2007a et 2007b. 
«fecte et passee soubz le scel de la prevosté* de Paris» devant «Pierre Chouart et Anthoyne Gouppil, notaires du roy nostre sire en son Chastellet de Paris» (lignes 51-54):

«Messire Nicole» a désormais le pouvoir « de traicter et appoincter avec ledits Choynet et sa femme d'un procés et descord [...] touchant la pocession ou propriété dudit fief de Clipponville» (lignes 54-57).

La procédure extrajudiciaire s'accélère: en moins d'un mois, les deux procureurs aboutissent à une transaction qui règle tous les points litigieux. Nicole Lecomte présente la proposition des Flavy (13 lignes), suit la réponse de Choinet en contrepartie ( 5 lignes) et la conclusion de l'accord par «messire Nicole» (4 lignes).

Commençons par l'offre faite par «messire Nicole» aux Choinet: le procureur du «chevalier et dame», qui a manifestement l'initiative du règlement du conflit - au motif de la prééminence de sa procuration tenue de la prévôté de Paris (lignes 61-62)? -, expose «de son bon gré» que les «droitz et tiltres desdits Choynet et sa femme» annulent «les droitz et tiltres d'iceulx chevalier et dame» (lignes 58-61). L'affirmation peut surprendre car toute l'histoire du procès devant les assises de Caudebec et devant la cour de l'Échiquier montrait que les deux parties ne pouvaient s'entendre faute de preuves («le principal de ladicte matiere n'eust peu bonnement estre congneu», lignes 25). Il semble que les Choinet ont pu présenter les documents nécessaires, conservés par les descendants de Michel Dumont, «tant par lectres, escroes ${ }^{\star}$, pappiers de recepte que autres escriptures touchant ledit fief» (lignes 59-60), on appréciera au passage la précision du tabellion quant aux différents types d'écrits ${ }^{92}$. On sait grâce aux registres du tabellionage que Michel Dumont et son gendre, Pierre Choinet, n'ont pas l'habitude de traiter leurs affaires avec désinvolture ${ }^{93}$. Sans doute avaient-ils des clercs en charge de conserver tous ces papiers, les tabellions n'avaient pas le monopole de la mémoire écrite des patrimoines.

«Messire Nicole Lecomte» annonce donc que «soy faisant fort, tant en son propre et privé nom, que en nom et comme procureur dudit chevalier », il apportera «lectres suffisantes à ses despens ausdits Choynet et sa femme dedens Noël prouchain venant» (lignes 62-65), c'est-à-dire avant le 25 décembre 1464. Les Flavy renoncent ainsi de "pretendre, demander et reclamer tant en petitore que pocessore» (lignes 68-69). En raison de leur propriété désormais admise, les Choinet toucheront tous les revenus, ceux de l'occupation (1419-1450) et ceux qui dataient du séquestre du fief (1449/50-1464). Le procureur Nicole Lecomte excepte pourtant les revenus que Flavy auraient pu toucher avant la mort de Robert Le Vigneron (lignes 75-78) - on appréciera le conditionnel qui nous apprend incidemment que le séquestre des revenus n'a pas été total depuis la

92. Bretthauer, 2019, p. 123-132.

93. SCORdia, 2019, vol. 1, $1^{\text {re }}$ partie (Pierre Choinet, "Un Normand du Pays de Caux»), p. $33-87$. 
clameur de haro et qu'il y avait eu certains arrangements entre Flavy et le premier mari de Jehanne Dumont. Nicole Lecomte envisage tous les cas pour éviter une reprise du conflit. Un accord impliquant de contenter les deux parties, Nicole Lecomte s'applique à présenter une solution que le chevalier et sa femme aient «agreable» (ligne 63).

De son côté, Pierre Choinet donne "cent escus d'or» aux Flavy (lignes 81-82). C'est la deuxième partie de l'accord. Chacun apporte ce qu'il a et chacun cherche à obtenir ce qui lui manque: les Flavy ont des fiefs nobles - manquaient-ils de liquidités?; les Choinet ont de l'argent et ils ambitionnent de posséder des terres nobles. Aussi, Pierre Choinet, procureur pour lui et pour sa femme, décide-t-il de verser 100 écus d'or, "presentement" réglés à Nicole Lecomte "pour et au nom desdits chevalier et dame» (lignes 81-82). Le futur médecin et astrologue de Louis XI agit "pour fuyr à la longueur du procés» (ligne 79), qui a un coût, tous sont d'accord sur ce point. Choinet ajoute qu'il baille les 100 écus d'or «pour aucunement [nullement] supporter aux frais, mises* [frais de justice] et despens fais par ledit chevalier en la poursuite d'icelui» (lignes 80-81). Choinet fait pourtant un autre geste financier puisqu'il annonce qu'il va payer l'intégralité de l'amende du haro «en quoy ils ont esté mis en la court dudit Eschiquier à cause dudit procés» (lignes 83-85).

En raison des offres des deux parties, le procureur Nicole Lecomte « delivra presentement ausdits Choynet pour lui et sa femme plusieurs lectres, escroes, pappiers, procés et escriptures que lesdits chevalier et dame avoient touchant icelui fief » (lignes 86-88). Là encore, notons que la documentation qui semblait manquante est maintenant retrouvée et remise aux nouveaux propriétaires de Cliponville. L'accord est garanti par Nicole Lecomte en raison de sa procuration et par les Flavy sur «tous les biens meubles et heritages desdits chevalier et dame» (lignes 90-91), et authentifié par les tabellions de Rouen en présence des deux témoins, comme il se doit.

Les deux familles Flavy et Choinet ont montré leur capacité à trouver des solutions grâce à des intermédiaires (procureurs et conseillers). Après la clameur de haro, les instances judiciaires ont rempli leur fonction par les enquêtes, les «veues» lancées pour vérifier les droits de chacun; le lieutenant du bailli a porté un jugement, un des plaignants a posé une doléance; la cour de l'Échiquier a ensuite émis un arrêt et renvoyé l'affaire aux assises. Le compromis de 1464, conservé aux Archives départementales de Seine-Maritime, fait manifestement comprendre que les parties sont lasses et cherchent à «eviter» un nouveau procès et à «fuyr à la longueur d'icelui procés». La longueur, il n'est pas dit la lenteur, et le coût de la justice font partie des critiques cent fois répétées dans les ordonnances et la littérature. La perception des procédures judiciaires se transforme au cours de l'affaire, bénéfiques au début, elles sont plus problématiques à la fin: l'annonce de la tenue d'un nouveau procès devant les assises de Caudebec apparaissant en fin de compte non comme une solution, mais une menace qui incite les parties à régler le conflit par une action infrajudiciaire, en dehors des assises du bailliage et la cour de l'Échiquier grâce aux officiers royaux du tabellionage. 
Ainsi l'affaire Flavy-Choinet, telle qu'elle est présentée dans l'acte de tabellionage, apporte des informations sur les procédures sollicitées en cas de conflit et sur le vocabulaire des tractations juridiques. Le bilan financier de l'acte atteste que les Choinet ont des liquidités, l'importante somme, dont il faut noter qu'elle n'est pas donnée en monnaie de compte (livres tournois) mais en monnaie de règlement, correspond à une sorte de dédommagement forfaitaire couvrant une partie de la valeur de Cliponville, les revenus du fief depuis la clameur de haro, le règlement de l'amende, sans parler des frais de justice. Sur le plan patrimonial, le procès a été suivi et réglé grâce à Pierre Choinet, le second mari de Jehanne Dumont, au droit de sa femme et de ses héritiers. L'acte du 20 mai 1464 fait entrer le fief noble de Cliponville dans le patrimoine de Thomas Le Vigneron, le fils de Jehanne Dumont et de Robert Le Vigneron, son premier mari. Dans un acte bien postérieur au conflit, Thomas est effectivement titré seigneur de Cliponville ${ }^{94}$. Le bilan social complète l'approche patrimoniale, l'argumentation de la paix et de l'amour exposé comme le motif du règlement du procès n'est pas un lieu commun. À l'échelle royale, elle répond aux instructions de Charles VII de pacifier la Normandie après le départ des Anglais, c'est un idéal à atteindre; à l'échelle locale, elle fait partie des relations obligées et nécessaires entre des familles du Pays de Caux, amenées à se côtoyer. La "paix et l'amour nourrir entr'eulx» constituent le soubassement du lien social et la condition de la remise en valeur de la rive droite de la Seine.

\section{Conclusion}

Les diverses informations extraites de l'acte du 20 mai 1464 prouvent à l'évidence la richesse du tabellionage normand, ici plus spécifiquement rouennais. Grâce à un seul acte ( 56 lignes), il est possible de reconstituer l'histoire d'un conflit entre deux familles avant, pendant et après la monarchie lancastrienne. L'annexe 4 donne la liste des différents acteurs (16) ayant contribué de près ou de loin à l'affaire. Les registres gardent la mémoire des procédures (clameur de haro, assises de Caudebec, cour de l'Échiquier) et permettent d'appréhender le règlement d'un conflit, alors que ce type de compromis est souvent mal connu puisqu'ayant échappé aux archives judiciaires. Les tabellions rappellent la nature des liens (mariage, filiation) des actants et n'omettent pas de mettre en avant la coutume normande, qui a pour but de conserver les patrimoines, ici par héritières interposées. Jehanne Dumont est l'une d'entre elles, qui se trouve à la tête de biens hérités de son père Michel Dumont et de sa mère Maline Malleterre. Ces biens ne passent aucunement dans le patrimoine de ses deux maris. Si Robert Le Vigneron comme Pierre Choinet actent, ils le font «au droit» et en présence de Jehanne Dumont. Les stratégies matrimoniales

94. Thomas Le Vigneron, écuyer, sieur de Cliponville, seul fils et héritier de Jehanne Dumont, Charles de BeAurePaIre, 1906, p. 285. Il n'apparaît plus à Cliponville dans l'information commandée par Louis XII en 1503, Registre des fiefs et arriere-fiefs du bailliage de Caux en 1503, fiefs et arrière-fiefs du bailliage de Caux en 1503, A. Beaucousin (éd.) 1891, p. 207. 
ont fait en sorte de marier Jehanne à des Rouennais gradués en droit, capables de supporter les procès en cours. C'est finalement Pierre Choinet, fort habile homme, omniprésent dans l'acte (lignes 17, 31, 47, 55, 56, 65, 72, 77, 79, 82, 83, 86, 89), qui parvient à un accord passé devant les tabellions de Rouen grâce à l'autorité de Nicole Lecomte ${ }^{95}$, le procureur des Flavy, qui a su proposer au futur médecin de Louis XI un arrangement qui contente les deux parties. Des négociations orales ont dû avoir lieu entre les procureurs pour discuter chaque point ${ }^{96}$, elles ont abouti à l'accord du 20 mai.

L'affaire Flavy-Choinet n'est qu'un exemple des procès en cours dans les années 1450-1460 entre Jehanne Dumont, l'héritière des biens obtenus par son père sous la monarchie lancastrienne, et les anciens propriétaires. Il ne s'agit nullement de généraliser l'histoire de la pacification cauchoise à partir d'une seule transaction. L'analyse d'autres affaires ayant opposé les Choinet à des familles nobles tend plutôt à prouver que chacune s'est terminée par une résolution différente. Les biens acquis par Michel Dumont, tantôt sont restés à ses descendants moyennant finances (affaire Flavy-Choinet), tantôt sont retournés à leurs propriétaires ante bellum (affaire Clères-Choinet), tantôt ont été échangés contre un autre bien (affaire Craon-Choinet) ${ }^{97}$. Chaque fois, les procédures judiciaires n'ont pu régler les conflits et des compromis infrajudiciaires ont été passés devant un tabellion.

Les registres du tabellionage témoignent des changements de propriété dans la Normandie lancastrienne, ils gardent la mémoire des moyens mis en œuvre pour trouver des solutions à l'échelle locale, non seulement acceptables par chaque famille mais porteuses de transactions futures ${ }^{98}$.

95. On aimerait en savoir un peu plus sur cet acteur de la pacification en Pays de Caux.

96. «Résoudre les conflits relève de la négociation plus que de décisions irréversibles ", GAUVARD, 2001, p. 372.

97. SCORDIA, 2019, vol. 1, p. 63-73

98. Cf. supra note 42. Les changements de propriété dans le duché de Normandie ont eu des conséquences sociales et financières. Dès 1461, Louis XI avait prescrit une information générale de la noblesse en Normandie pour distinguer ceux qui se prétendaient nobles du fait de l'acquisition de fiefs nobles et leur faire payer le droit de franc-fief. L'information a mené à la charte générale des francs-fiefs ( 5 novembre 1470, ORF, t. 17, p. 337-341), spécifique à la Normandie. La charte aboutit à l'anoblissement des possesseurs des fiefs nobles et de ceux qui en acquerraient d'ici 40 ans. Reulos, 1996, p. 741-742 (discussion lors des $23^{\mathrm{e}}$ Journées d'histoire du droit et des institutions des Pays de l'Ouest de la France, Caen, 22 et 23 mai 1995). On trouve ainsi le nom de Thomas Le Vigneron, demeurant à Rouen, qui acquitte 15 livres tournois (sergenterie de Pavilly), Arch. dép. Seine-Maritime, F 38, Monfault, «Recherche de la noblesse en Normandie par Monfault en l'an 1469 ", s.l.n.d, 26 pages manuscrites, voir la partie intitulée «Recepte de la vicomté de Rouen sur les personnes à ce contribuables qui ont esté ennoblis par charte de francs fiefs selon la charte de l'an 1470 ». Et pour la période postérieure, lire PoIrEY, 2011. 


\section{Bibliographie}

\section{Sources}

L'Ancienne Coutume de Normandie, William-Laurence de Gruchy (éd.), Saint-Hélier (Jersey), Charles Le Feuvre, 1881 (Grand Coutumier en latin avec version française en regard).

Arresta communia Scacarii. Deux collections d'arrêts notables de l'Échiquier de Normandie de la fin du XIII e siècle (1276-129o, 1291-1294), Ernest Perrot (éd.), Caen, L. Jouan, Bibliothèque d'histoire du droit normand, 1910.

Coutumiers de Normandie, Ernest-Joseph Tardif (éd.), Rouen-Paris, Lestringant / Picard, 1881-1903, 2 t. en 3 vol.: Très ancien Coutumier, t. 1, vol. 1 (texte latin); t. 1, vol. 2 (textes français et normand); Summa de legibus in curia laicali, t. 2.

[Enguerran de Monstrelet], La chronique d'Enguerran de Monstrelet, en deux livres, avec pièces justificatives, 1400-1444, Louis Douët d'Arcq (éd.), Paris, Renouard, SHF, 1857-1862, 6 vol.

Gilles le Bouvier, dit le Héraut Berry, Les chroniques du roi Charles VII, Henri Courteault et Léonce Celier (éd.) avec la collaboration de Marie-Henriette Jullien de Pommerol, Paris, Librairie C. Klincksieck, SHF, 1979.

Pierre Choinet, Le Livre des trois âges, Lydwine Scordia (éd.), Rouen, Presses universitaires de Rouen et du Havre (PURH), 2009.

Pierre Choinet, Le Rosier des guerres, Lydwine Scordia (éd.), à paraître à la SHF.

Recherche de Montfaut contenant les noms de ceux qu'il trouva nobles et de ceulx qu'il imposa à la taille quoiqu'ils se prétendissent nobles en l'année 1463, P. Labbey de La Roque (éd.), Caen, Impr. F. Poisson, 1818.

Registre des fiefs et arrière-fiefs du bailliage de Caux en 1503, A. Beaucousin (éd.), Rouen, A. Lestringant, 1891.

\section{Bibliographie}

Allmand, Christopher T., «Documents relating to the Anglo-French Negociations of 1439", Camden Miscellany, 24, 1972, p. 79-149.

Angers, Denise, Le terrier de la famille d'Orbec à Cideville (Haute-Normandie) XIV $X V I^{e}$ siècles, préface d'Henri Dubois, Montréal, Les Presses de l'Université de Montréal, 1993.

ANGERs, Denise, «Libertés et contrainte dans les actes de femmes dans le tabellionage de Caen $\left(2^{\mathrm{e}}\right.$ moitié du XV ${ }^{\mathrm{e}}$ siècle) $)$, Tabularia, Les femmes et les actes, 2003, DOI: 10.4000/tabularia.1559.

Les Anglais en Normandie, Actes $d u 45^{e}$ Congrès organisé par la Fédération des Sociétés historiques et archéologiques de Normandie, Saint-Sauveur-le-Vicomte, 20-24 octobre 2010, Bernard Bodinier (dir.), Louviers, Fédération des Sociétés historiques et archéologiques de Normandie, 2011.

L'arbitrage, Anne Lefebvre-Teillard, Marie-Anne Cohendet et Renaud Denoix De Saint Marc (dir.), Paris, Dalloz, Archives de philosophie du droit, 52, 2009.

Tabularia «Etudes», «Richesse du tabellionage normand...», 2020, p. 1-38, 22 juillet 2020 
Arnoux, Mathieu, «De la charte à l'acte de tabellion. Formes locales, régionales ou nationales d'une transition. Réflexions à partir du cas normand», in Tabellions et tabellionages de la France médiévale et moderne, Mathieu Arnoux et Olivier Guyotjeannin (dir.), Paris, École nationale des chartes, Mémoires et documents de l'École des Chartes, 90, 2011, p. 7-27.

Beaune, Colette, Naissance de la nation France, Paris, Gallimard, 1985.

Beaurepaire, Charles de, «Notice sur Pierre Choynet à propos de son inscription funéraire conservée à Monville», Bulletin de la Commission des antiquités de la Seine-Inférieure, 13, 1903 à 1905, 1906, p. 281-292.

BESNIER, Robert, "Le procès possessoire dans le droit normand du XII ${ }^{e}$ et du XIII ${ }^{\mathrm{e}}$ siècle», Revue historique de droit français et étranger, 31, 1953, p. 378-408.

BESNIER, Robert, "Actualité de la clameur de haro dans le droit de l'île de Guernesey», Droit privé et institutions régionales. Études historiques offertes à Jean Yver, Paris, PUF, 1976, p. 63-68.

Billoré, Maïté, Mathieu, Isabelle et Avignon, Carole, La justice dans la France médiévale (VIII $-X V^{e}$ siècle), Paris, Armand Colin, 2012.

de Boos, Emmanuel, Damongeot, Marie-Françoise, Roger, Jean-Marc et Vielliard, Françoise, préface de Michel Pastoureau, L'Armorial Le Breton, Paris, Archives nationales-Groupe Malakoff-Somogy, 2004.

Bouckard, François, Guide des archives de la Seine-Maritime, t. 1. Généralités. Archives antérieures à 1790, Rouen, Archives départementales, 1990.

BRETTHAUER, Isabelle, «Le statut du registre entre usage privé et usage public», in Tabellionages au Moyen Âge en Normandie. Un notariat à découvrir, Jean-Louis Roch (dir.), Rouen, Presses universitaires de Rouen et du Havre (PURH), 2014, Changer d'époque, 28, p. 13-24.

Bretthauer, Isabelle, «Les transactions par ceux qui les font. Perception professionnelle de la transaction $\left(\mathrm{XIV}^{\mathrm{e}}-\mathrm{XV}^{\mathrm{e}}\right.$ siècle)», in Transiger. Éléments d'une ethnographie des transactions médiévales, Julie Claustre (dir.), Paris, Éditions de la Sorbonne, 2019, p. 123-152, DOI: 10.400o/books.psorbonne.54713.

CAILlEux, Philippe, «La présence anglaise dans la capitale normande: quelques aspects des relations entre Anglais et Rouennais", in La Normandie et l'Angleterre au Moyen Âge, Pierre Bouet et Véronique Gazeau (dir.), Caen, Publications du CRAHM, 2003, p. 265-276.

CaIlleux, Philippe, Trois paroisses de Rouen, XIII ${ }^{e}-X V^{e}$ siècles: Saint-Lô, Notre-Damela-Ronde et Saint-Herbland. Étude de topographie et d'urbanisme, Rouen-Caen, Presses universitaires de Rouen et du Havre (PURH) / Presses universitaires de Caen, 2011a, DOI : 10.400o/books.purh.10691.

CaIlleux, Philippe, «Tabellions et tabellionages de Rouen et de sa vicomté (XIV e$\mathrm{XV}^{\mathrm{e}}$ siècles)", in Tabellions et tabellionages de la France médiévale et moderne, Mathieu Arnoux et Olivier Guyotjeannin (dir.), Paris, École nationale des chartes, Mémoires et documents de l'École des Chartes, 90, 2011b, p. 155-178.

CAIlleux, Philippe, «Pratiques et tarifications des actes des tabellions rouennais à la fin du Moyen Âge», in Tabellionages au Moyen Âge en Normandie. Un notariat à découvrir, Actes de la Journée d'étude du GRHIS (14 novembre 2012), Jean-Louis Roch (dir.), Rouen, Presses universitaires de Rouen et du Havre (PURH), Changer d'époque, 24, 2014, p. 25-44. 
CARrIER, Nicolas, «Une justice pour rétablir la "concorde": la justice de composition dans la Savoie de la fin du Moyen Âge (fin XIII ${ }^{\mathrm{e}}$-début XVI ${ }^{\mathrm{e}}$ siècle) », in Le règlement des conflits au Moyen Âge, Actes du $31^{\mathrm{e}}$ congrès de la Société des historiens médiévistes de l'enseignement supérieur public (Angers, 2000), Paris, Publications de la Sorbonne, 2001, p. 237-257, DOI : 10.3406/shmes.2000.1793.

Champion, Pierre, Guillaume de Flavy, capitaine de Compiègne. Contribution à l'histoire de Jeanne d'Arc et à l'étude de la vie militaire et privée au XV siècle, Paris, Honoré Champion, 1906.

Contamine, Philippe, «Charles VII, les Français et la paix, 1420-1445", Comptes rendus des séances de l'Académie des Inscriptions et Belles-Lettres, 137 / 1, 1993, p. 9-23. URL: https://www.persee.fr/doc/crai_oo65-0536_1993_num_137_1_15178.

Contamine, Philippe, «Rendre grâces, prier, faire mémoire: la "fête du roi”, 14 octobre de l'année 1450 puis 12 août de l'année 1451 et des années postérieures", Bulletin de la Société nationale des Antiquaires de France, 2009, 2012, p. 338-353. URL: https:// www.persee.fr/doc/bsnaf_oo81-1181_2012_num_2009_1_11105.

Curry, Anne, «Les villes normandes et l'occupation anglaise: l'importance du siège de Rouen (1418-1419)", in Les villes normandes au Moyen Âge, Pierre Bouet et François Neveux (dir.), Caen, Presses universitaires de Caen, 2006, p. 109-124. DOI: $10.4000 /$ books.puc.9449.

du Fresne de Beaucourt, Gaston, Histoire de Charles VII, Paris, Société bibliographique, 1881-1891, 6 vol.

Gauvard, Claude, "De grace especial”. Crime, État et société en France à la fin du Moyen Âge, Paris, Publications de la Sorbonne, 1991, 2 vol.

Gauvard, Claude, «Entre justice et vengeance: le meurtre de Guillaume de Flavy et l'honneur des nobles dans le royaume de France au milieu du $\mathrm{XV}^{\mathrm{e}}$ siècle», in Guerre, pouvoir et noblesse au Moyen Âge. Mélanges en l'honneur de Philippe Contamine, Jacques Paviot et Jacques Verger (dir.), Paris, Presses de l'université de Paris-Sorbonne, Cultures et civilisations médiévales, 22, 2000, p. 291-311.

Gauvard, Claude, "Conclusion", in Le règlement des conflits au Moyen Âge, Actes du $31^{\mathrm{e}}$ congrès de la Société des historiens médiévistes de l'enseignement supérieur public (Angers, 2000), Paris, Publications de la Sorbonne, 2001, p. 369-391.

GLasson, Ernest Désiré, «De la possession et des actions possessoires au Moyen Âge», Nouvelle revue d'histoire de droit français et étranger, 14, 189o, p. 588-633.

Guyotjeannin, Olivier, «Tabellions et tabellionages dans la France septentrionale. L'enquête du côté de la diplomatique médiévale», in Tabellions et tabellionages de la France médiévale et moderne, Mathieu Arnoux et Olivier GuyotjeAnnin (dir.), Paris, École nationale des chartes, Mémoires et documents de l'École des Chartes, 90, 2011, p. 29-48.

Hellot, Amédée, Essai sur les baillis de Caux de 1204 à 1789, avec documents inédits à l'appui, Paris, E. Dumont, 1895.

JeAY, Claude, «Les seings manuels des tabellions (Normandie, $\mathrm{XIV}^{\mathrm{e}}-\mathrm{XV}^{\mathrm{e}}$ siècles). Signes personnels ou expression du pouvoir royal?», in Tabellions et tabellionages de la France médiévale et moderne, Mathieu Arnoux et Olivier Guyotjeannin (dir.), Paris, École nationale des chartes, Mémoires et documents de l'École des Chartes, 9o, 2011, p. 325-348. 
LABrosse, Henri, «Un tarif du tabellionage de Rouen au XV $\mathrm{XV}^{\mathrm{e}}$ siècle», Bulletin de la Société de l'histoire de Normandie, 13, 1919-1924, p. 234-240.

LARDIN, Philippe, "La crise monétaire de 1420-1422 en Normandie», L'argent au Moyen Âge, Actes du XVIII ${ }^{\mathrm{e}}$ congrès de la Société des historiens médiévistes de l'enseignement supérieur public, Paris, Publications de la Sorbonne, Série Histoire ancienne et médiévale, 51, 1998, p. 101-143.

LARDIN, Philippe, «Dieppe sous la domination anglaise (1419-1435)», in Les Anglais en Normandie. Actes $d u 45^{e}$ Congrès organisé par la Fédération des Sociétés historiques et archéologiques de Normandie (Saint-Sauveur-le-Vicomte, 20-24 octobre 2010), Bernard Bodinier (dir.), Louviers, Fédération des Sociétés historiques et archéologiques de Normandie, 2011, p. 173-184.

LeCordier, Jimmy, «Mobilité des gens de garnison en Normandie lancastrienne, l'exemple de Caen (1423-1448)", in Les Anglais en Normandie. Actes du $45^{e}$ Congrès organisé par la Fédération des Sociétés historiques et archéologiques de Normandie (Saint-Sauveur-le-Vicomte, 20-24 octobre 2010), Bernard BodINIER (dir.), Louviers, Fédération des Sociétés historiques et archéologiques de Normandie, 2011, p. 205212.

Lefèvre-Pontalis, Eugène, «Épisodes de l'invasion anglaise: la guerre des partisans dans la Haute-Normandie (1424-1429) ", Bibliothèque de l'École des chartes, 54, 1893, p. $475-521 ; 55,1894$, p. 259-305; 56, 1895, p. 433-508.

Lemonnier-Lesage, Virginie, Le statut de la femme mariée dans la Normandie coutumière: droit et pratiques dans la généralité de Rouen, préface Yves Sassier, Clermont-Ferrand, Presses universitaires de droit, 2005.

Lemonnier-Lesage, Virginie, «Les tabellions et l'assouplissement de la norme», in Tabellions et tabellionages de la France médiévale et moderne, Mathieu Arnoux et Olivier Guyotjeannin (dir.), Paris, École nationale des chartes, Mémoires et documents de l'École des Chartes, 90, 2011, p. 349-366.

LemonNiER-Lesage, Virginie, «Les tendances communautaires des époux à la lecture des actes des tabellions et des notaires dans la Normandie coutumière de l'époque moderne", in Tabellionages au Moyen Âge en Normandie. Un notariat à découvrir, Actes de la Journée d'étude du GRHIS (14 novembre 2012), Jean-Louis RocH (dir.), Rouen, Presses universitaires de Rouen et du Havre (PURH), Changer d'époque, 24, 2014, p. 67-81.

Maneuvrier, Christophe, "Des sociétés rurales entre droit, pratique et procès ", Histoire et Société Rurales, 28, 2007a, p. 7-10.

Maneuvrier, Christophe, «L'activité d'un notaire de la fin du XIV e siècle: à propos du registre de Guillaume Guérart, tabellion de Lisieux (1390-1393)», in Justice et gens de justice en Normandie, Actes du $41^{\mathrm{e}}$ congrès organisé par la Fédération des sociétés historiques et archéologiques de Normandie, Saint-Pierre-sur-Dives, 12-15 octobre 2006, Bernard Bodinier (éd.), Louviers, Fédération des Sociétés historiques et archéologiques de Normandie, 2007b, p. 125-131.

Mollat, Michel, «Un "collaborateur” au temps de la guerre de Cent Ans: Jehan Marcel, changeur à Rouen", Annales. Histoire, Science Sociales, 1, 1946/1, p. 36-42.

MusseT, Jacqueline, Le régime des biens entre époux en droit normand du XVI siècle à la Révolution française, Caen, Presses universitaires de Caen, 1997. 
Neveux, François, «La constitution d'un réseau urbain en Normandie», in Les villes normandes au Moyen Âge, Pierre Bouet et François Neveux (dir.), Caen, Presses universitaires de Caen, 2006, p. 45-60.

Neveux, François, «Le contexte historique de la rédaction des coutumiers normands », Annales de Normandie, 61, 2011/2, p. 11-22.

Pigorneau, Vincent, «Le siège de Rouen vu à travers un registre de tabellionage en 1418 », mémoire de maîtrise, Philippe Lardin (dir.), Université de Rouen, 1997.

PoIrey, Sophie, «Le droit normand d'après 1204: ruptures et continuités ", in 1204. La Normandie entre Plantagenêts et Capétiens, Anne-Marie FlambardHéricher et Véronique Gazeau (dir.), Caen, Publications du CRAHM, 2004, p. 289-308.

PoIrey, Sophie, «Approches juridiques de la noblesse dans la coutume de Normandie», in Les noblesses normandes (XVI ${ }^{e}-X I X^{e}$ siècle), Alain HUgON et Ariane Bolt ANSKI (dir.), Rennes, Presses universitaires de Rennes (PUR), 2011, p. 63-77. DOI: 10.400o/books.pur.120168.

Poumarède, Jacques, «La protection possessoire dans les coutumes du Sud-Ouest de la France au Moyen Âge», Droit privé et institutions régionales. Études historiques offertes à Jean Yver, Rouen, Presses universitaires de Rouen et du Havre (PURH), Paris, PUF, 1976, p. 595-604. DOI: 10.400o/books.purh.12572.

Reulos, Michel, «"Anobli aux francs-fiefs”: origine et portée de l'expression », Annales de Normandie, 46/5, 1996, p. 741-742.

Roch, Jean-Louis, «Introduction", in Tabellionages au Moyen Âge en Normandie. Un notariat à découvrir, Actes de la Journée d'étude du GRHIS (14 novembre 2012), Jean-Louis Roch (dir.), Rouen, Presses universitaires de Rouen et du Havre (PURH), Changer d'époque, 24, 2014a, p. 9-10. DOI: 10.400o/books.purh.1472.

Roch, Jean-Louis, "Conclusion. Tabellionages, mode d'emploi», in Tabellionages au Moyen Âge en Normandie. Un notariat à découvrir, Actes de la Journée d'étude du GRHIS (14 novembre 2012), Jean-Louis Roch (dir.), Rouen, Presses universitaires de Rouen et du Havre (PURH), Changer d'époque, 24, 2014b, p. 139-144.

Roch, Jean-Louis, «Femmes et métiers dans la région rouennaise au Moyen Âge», in "Tout ce qu'elle saura et pourra faire". Femmes, droits, travail en Normandie du Moyen Âge à la Grande Guerre, Anna Bellavitis, Virginie Jourdain, Virginie Lemonnier-Lesage et Béatrice Zucca Micheletto (dir.), Rouen, Presses universitaires de Rouen et du Havre (PURH), 2015, p. 21-28.

SAdourny, Alain, "L'occupation anglaise en Haute-Normandie (1417-1449)", in Les Anglais en Normandie. Actes du $45^{e}$ Congrès organisé par la Fédération des Sociétés historiques et archéologiques de Normandie (Saint-Sauveur-le-Vicomte, 20-24 octobre 2010), Bernard Bodinier (dir.), Louviers, Fédération des Sociétés historiques et archéologiques de Normandie, 2011, p. 165-171.

SCORDIA, Lydwine, «Devoir de justice et amour du roi aux $\mathrm{XIII}^{\mathrm{e}}-\mathrm{XV}^{\mathrm{e}}$ siècles », in $L e$ roi fontaine de justice. Pouvoir justicier et pouvoir royal au Moyen Âge et à la Renaissance, colloque international d'Orléans, 1-2 juillet 2010, Silvère MENEGALDo et Bernard RibÉmont (dir.), Paris, Klincksieck, 2012, p. 129-143.

Scordia, Lydwine, Pierre Choinet et le Rosier des guerres, mémoire inédit, 2 vol., in L'argent, l'amour et la mort (XIII ${ }^{e}-X V^{e}$ siècle), HDR, Université de Nanterre Paris Ouest, 2019, 4 vol. 
Sion, Jules, Les paysans de la Normandie orientale: Pays de Caux, Bray, Vexin Normand, Vallée de la Seine. Étude géographique, Paris, Armand Colin, 1909.

TAввAGH, Vincent, «Famille et société: l'exemple de Rouen au XV $\mathrm{XV}^{\mathrm{e}}$ siècle», Villes et sociétés urbaines au Moyen Âge. Hommage à M. le Pr Jacques Heers, Paris, Presses de l'université de Paris Sorbonne, 1994, p. 249-258.

Tabellions et tabellionages de la France médiévale et moderne, Mathieu ArNoux et Olivier Guyotjeannin (dir.), Paris, École nationale des chartes, Mémoires et documents de l'École des Chartes, 90, 2011.

Tabellionages au Moyen Âge en Normandie. Un notariat à découvrir, Actes de la Journée d'étude du GRHIS (14 novembre 2012), Jean-Louis Roch (dir.), Rouen, Presses universitaires de Rouen et du Havre (PURH), Changer d'époque, 24, 2014.

"Tout ce qu'elle saura et pourra faire". Femmes, droits, travail en Normandie du Moyen Âge à la Grande Guerre, Anna Bellavitis, Virginie Jourdain, Virginie Lemonnier-Lesage et Béatrice Zucca Micheletto (dir.), Rouen, Presses universitaires de Rouen et du Havre (PURH), 2015.

VAUTIER, Charles, Extrait du registre des dons confiscatoires maintenus et autres actes faits dans le duché de Normandie pendant les années 1418, 1419 et 1420 par Henri V, roi d'Angleterre, Paris, A Lord Byron, 1828. 


\section{Annexes}

\section{Annexe 1 - Transcription de l'acte passé le 20 mai 1464 devant le tabellionage de Rouen (Arch. dép. Seine-Maritime, 2 E 1/193, clichés 97-98) ${ }^{99}$}

1 Comme ja pieça, procés se feust meu es assises ${ }^{\star}$ de Caudebec entre noble homme, messire

2 Ector de Flavy, chevalier, et madame Marie de Calleville, sa femme, au droit d'elle ${ }^{\star}$, d'une

3 part, et Thomas Coulombel et Maline, sa femme, par avant femme de deffunct Michel Dumont,

4 d'autre part, à cause et pour raison d'un fief ou porcion de fief noble assis à Clipponville et

5 environ, que chacune desdictes parties disent à soy appartenir à certains tiltres; et auquel fief,

6 icelui de Flavy, au droit dessudit, se fust mis en pocession dedens l'an de la redducion

7 [soumission] et reboutement [expulsion] des Angloys de ce pays et duchié de Normandie, dist

8 que ses predecesseurs en avoit joy es dernieres annees precedens la venue et descente desdits

9 Angloys oudit pays, qu'ilz n'avoient peu entretenir à cause de ladicte descente et occupacion

10 fecte par lesdits Angloys dudit pays, et partant feust retourné en icelle dedens l'an de ladicte

11 redducion, laquelle pocession lui fust lors empeschee par clameur de haro* ${ }^{\star}$, fecte par Guillebert

12 Couppequesne pour et en nom desdits Coulombel et sa femme, dont ledit procés feust meu et

13 pendant $^{\star}$ esdictes assises et veue ${ }^{\star}$ termees [fixer qqc à une date donnée] entr'eulx.

14 Lequel procés eust esté depuis recueilli [supporter, DMF.I.A.2.e] par deffunt Robin Le

15 Vigneron et Jehanne Dumont, sa femme, au droit d'elle, comme heritiere de deffunct Colin

16 Dumont, son frere, filz et heritier dudit deffunct Michel Dumont. Et, depuis le trespas dudit Le

17 Vigneron, derechief recueilli par honnorable maistre Pierre Choynet, docteur en medicine, et

18 ladicte Jehane Dumont, à present sa femme. Sur quoy, eust esté tant procedé que la veue eust

19 esté fecte, et tenue et plaidee esdictes assises le cas desdictes parties en la presence des gens de

20 ladicte veue, passée sans saon ${ }^{\star}$, en propoz, responce, repplique ${ }^{\star}$ et dupplique ${ }^{\star}$. Et se feussent

21 partant [par suite] ledictes parties mis en jugement de l'accord duquel, fait par Robinet Helart,

22 lieutenant general du bailli de Caux, ledit de Flavy eust prins dolleance* sortissant $^{\star}$ en

23 l'Eschiquier* de Normandie, à present seant à Rouen. Ouquel, aprez ce que lesdictes parties

24 oulrent faicte ouverture ${ }^{\star}$ de ladicte dolleance, la court, voyant que par ladicte procedure fecte

25 par lesdictes parties, le principal de ladicte matiere n'eust peu bonnement estre congneu, eust

26 mis ledits jugement et dolleance au neant et appoinctié [convenir] qu'ilz procederoient en la

27 matere en l'estat qu'ilz estoient ou temps dudit jugement plaidé.

99. L'acte a été décomposé par nos soins en sept paragraphes pour aider à saisir les étapes du compromis de l'affaire Flavy-Choinet. Le registre est désormais en ligne: http://recherche.archivesdepartementales76.net/?id=viewer\&doc=accounts\%2Fmnesys_ad 76\%2Fdatas\%2Fir\%2Fserie_E_ seigneuries_familles_tabellionnage_rouen\%2FFRADo76_IR_E_002E_tabellionage_et_notariat_ rouen\%2Exml\&page_ref=27397\&lot_num=1\&img_num=1\&index_in_visu. 
28 En ensuivant lequel appoinctement, lesdictes parties eussent plaidé leur matere en propoz, 29 response et repplique sur le pocessoire* dudit fief. Finablement, icelles parties comparens ${ }^{*}$, 30 c'est assavoir lesdits chevalier et dame par messire Nicole Lecomte, le procureur ${ }^{\star}$, d'une part, 31 et ledit maistre Pierre Choinet en personne, pour lui et procureur de sa dicte femme, d'autre 32 part, affin de savoir et congnoistre sommierement [complètement] et de plain^ auquel d'eulx 33 ledit fief appartient en principal, eussent accordé aller plaider esdictes assises de Caudebec 34 devant ledit bailli de Caux ou son lieutenant et illec termer veue sur le droit principal et 35 proprietaire dudit fief et que les deniers estant en sequestre* ${ }^{*}$ ar ledit haro, yssus de la revenue 36 dudit fief, feussent entr'eulx parties [répartir] et receuz par moictié; et oultre, que pendant le 37 procés, ilz joyssent semblablement par moictié des fruitz et revenues d'icelui fief par baillant 38 cauxion suffisant de restituer par celui qui decherroit ${ }^{*}$ ce qu' il aura receu, tant dudit sequestre 39 que desdictes revenues depuis escheues a celui qui obtiendroit en fin de cause; accordans aussi 40 que, ou cas que à la prouchaine assise, aprez ladicte veue fecte, la matere n'eust prinse fin et 41 qu'il y eust aucun delay prins par lesdictes parties plaidant leur matere en l'absence des gens 42 d'icelle veue, comme fere eussent peu en leur presence, parce que se ilz se feussent appointés 43 en preuve, leurs fais eussent esté escrips, et celui qui l'eust eue a fere l'eust fecte, ainsi qu'il 44 eust appartenu, et partant lesdictes parties eussent fecte l'amende* dudit haro par main 45 commune $^{*}$, veu lequel accord, ladicte cause eust esté par ladicte court renvoyee à la prouchaine 46 assise dudit lieu de Caudebec, jouxte l'arrest dudit Eschiquier sur ce fait. Et depuis ce, ledit 47 messire Nicole, procureur, dessus nomé pour ce, en nom que dessus, et ledit Choynnet et sa 48 femme, pour eviter à icelui procés et pour paix et amour nourrir entr'eulx, par le moyen de leurs

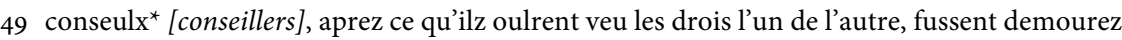
50 d'accord en la maniere qui s'ensuit:

51 Savoir faisons, fut present ledit messire Nicole Le-[cliché 98]-comte, lequel, en nom et comme 52 procureur dudit Ector de Flavy, ayant povoir par vertu de sa procuracion fecte et passee soubz 53 le scel de la prevosté ${ }^{\star}$ de Paris en ce present an mil CCCC LXIIII, le XXV jour d'avril, devant 54 Pierre Chouart et Anthoyne Gouppil, notaires du roy notre sire en son Chastellet de Paris, de 55 traicter et appoincter avec lesdits Choynet et sa femme d'un procés et descord meu et pendant 56 entre lesdits chevalier et dame d'une part, et lesdits Choynet et sa femme d'autre part, touchant 57 la pocession ou propriecté dudit fief de Clipponville, desquelles la teneur ensuit.

58 De son bon gré [de sa propre volonté], aprez ce qu'il lui fust apparu deuement ${ }^{*}$ des droitz et 59 tiltres desdits Choynet et sa femme, tant par lectres, escroes ${ }^{\star}$, pappiers de recepte que autres 60 escriptures, touchant ledit fief, preferans [passer avant, DMF, I.B] et adnullans les droitz et 61 tiltres d'iceulx chevalier et dame, par vertu et en usage du povoir à lui donné par ladicte 62 procuracion en tant que fere le povoit par icelle, et soy faisant fort, tant en son propre et privé 63 nom, que en nom et comme procureur dudit chevalier, que lesdits chevalier et dame auroient 64 agreable ce qui ensuit, et leur fera ratiffier, agre(e)r, et en apportera lectres suffisantes à ses 
65 despens ausdits Choynet et sa femme dedens Noel prouchain venant, congnut et confessa [faire 66 connaître qqc], pour et en nom desdits chevalier et dame, soy estre desisté et departi [renoncer $67 \grave{a}, D M F, I I I . A .4 . a]$ et encores se desiste et depart par ces presentes d'icelui procés, ensemble de 68 tout et tel droit que iceulx chevalier et dame s'aucun en avoient ou eussent peu avoir, pretendre, 69 demander et reclamer, tant en petitore ${ }^{\star}$ que pocessore à quelque droit, tiltre, raison ou moyen 70 que ce soit en icelui fief, terre et seigneurie de Clipponville, revenues appartenant et appendant 71 d'icelui quelzconques, et mesmes en toutes les levees et revenues dudit fief eschues, tant du 72 temps du sequestre dudit fief que autrement, voulant et accordant que lesdits Choynet et sa 73 femme en jouyssent come de leur propre chose sans ce que jamais iceulx chevalier et dame ne 74 leurs heritiers par quelque aultre voye ou moyen que ce soit y puissent mectre ne donner aucun 75 contredit [sans opposition] ou empeschemens pour le temps advenir, sauf que se lesdits 76 chevalier et dame avoient aucune chose tue ou receue du sequestre d'icelle terre escheu au 77 devant du trespas dudit Le Vigneron, lesdits Choynet et sa femme ne leur en pourroient aucune 78 chose demander.

79 Et par ce, icelui Choynet, pour lui et sadicte femme, pour fuyr à la longueur d'icelui procés et 80 pour aucunement supporter aux frais, mises ${ }^{*}$ et despens fais par ledit chevalier en la poursuite 81 d'icelui, donna ausdits chevalier et dame la somme de cent escus d'or, qui presentement furent 82 paiez et baillés par ledit Choynet audit messire Nicole pour et ou nom desdits chevalier et dame.

83 Dont et oultre, ledit Choynet pour lui et sadicte femme se submist, acquicta et delivra lesdits

84 chevalier et dame de l'amende en quoy ilz ont esté mis en la court dudit Eschiquier à cause 85 dudit procés.

86 Et, par ce moyen, ledit procureur bailla et delivra presentetement ausdits Choynet pour lui et sa 87 femme plusieurs lectres, escroes, pappiers, procés et escriptures que lesdits chevalier et dame 88 avoient touchant icelui fief; et se aucunes lectres et escriptures en peult recouvrer, les promist 89 semblablement bailler et delivrer à iceulx Choinet et sa femme, touteffois que recouvrer les 90 pourra. Le tout pour estre d'aussi ${ }^{100}$ comme elles estoient, oblige ledit messire Nicole par vertu 91 de sadicte procuracion et en tant que fere le peult par icelle tous les biens meubles et heritages 92 desdits chevalier et dame, presens Robinet Delamare et Robinet Le Caron.

100. Comprendre: d'aussi [grande force] comme elles estoient [auparavant]. 
Annexe 2 - Lexique judiciaire et infrajudiciaire de l'acte du 20 mai 1464

amende, - de main commune: amende payée solidairement par le demandeur et le défenseur (Dictionnaire du Moyen français, désormais DMF, s.v. «main", I.A.4.b). DMF Atilf: http://www.atilf.fr/dmf/.

assises: droit, session judiciaire (DMF, C.1.b); droit normand, «De assise» (L'Ancienne Coutume de Normandie, ch. LV, p. 140).

clameur de haro: coutume normande, sommation juridique de surseoir à toute entreprise et de se rendre immédiatement devant la justice pour faire statuer sur le différend ${ }^{101}$; "De haro» (L'Ancienne Coutume de Normandie, ch. LV, p. 136-140).

comparant, adj.: droit, qui comparaît en justice.

déchoir: droit, être débouté de qqc (DMF, B.2.c).

doléance, prendre -: droit, plainte en justice (DMF, B.3); Rem.: voie de recours devant une juridiction supérieure motivée par la mise en cause du juge inférieur.

droit, au - d'elle: droit normand, en raison de l'incapacité juridique de la femme et au motif de l'inaliénabilité de son patrimoine, le mari acte au droit de sa femme («De breif de mariage encombré», L'Ancienne Coutume de Normandie, ch. C, p. 243-244).

dûment, adv.: comme il convient, conformément à la loi (DMF, A).

duplique, subst.: droit, réponse à une réplique (DMF, II).

Échiquier: droit, Normandie, cour ducale (DMF, C.1.a); «De Eschiquier» (L'Ancienne Coutume de Normandie, ch. LVI, p. 141).

écrou : parchemin, écrit établi sous seing privé ou officiel, portant reconnaissance d'une dette, d'une obligation (DMF, B) ou registre, état de l'argent dépensé pour l'entretien de la maison du roi ou d'un grand seigneur (DMF, B.2.c).

mises: frais de justice (DMF, A.1).

ouverture, faire - : faire des propositions (DMF, B.3.a. Rem).

pendant, adj.: droit, d'un procès qui n'est pas réglé, qui est en instance (DMF, I.B).

pétitoire, action -: droit, action en revendication pour obtenir la restitution d'un bien en propriété; droit normand, «De possession non mouvable»; «De querelle fieffal »; «De brief de nouvelle dessaisine» (L'Ancienne Coutume de Normandie, ch. XCI, p. 211-212; ch. XCII, p. 212-214; XCIII, p. 214-220).

plain, sommairement et de -: droit: (procéder) directement, sans passer par le juge, sans observer les formalités ordinaires (DMF, III.B).

101. Besnier, 1976, p. 65-67; CAIlleuX, 2011a, p. 485. 
possessoire, action - : droit, action tendant à maintenir ou rétablir la possession considérée comme un état de fait distinct de la propriété (DMF, II.A); «De haro »; "De querelle de possession »; «De possession non mouvable» (L'Ancienne Coutume de Normandie, ch. LIV, p. 136-140; ch. LXXXVI, p. 198-201; ch. XCI, p. 211-212).

prévôté: à Paris, circonscription royale équivalente à un bailliage.

procureur : celui qui conduit les affaires (DMF).

réplique, subst.: droit, réponse à la partie adverse (DMF, B.2.a).

saon, sans - : sans reproche à l'égard des témoins (DMF, A).

séquestre: droit, dépôt d'une chose en litige aux mains d'une tierce personne en attendant le règlement de la contestation (DMF, B).

sortir juridiction : droit, subir le jugement (DMF, I.C.1.c).

veue: droit normand, instruction, interrogatoire, enquête sur place par témoins convoqués (DMF, D.2.b); «De veue» (L'Ancienne Coutume de Normandie, ch. LXVI, p. 154-156 et XCV, p. 221-230). 
Annexe 3 - Chronologie des procédures

judiciaires et infrajudiciaires de l'affaire Flavy-Choinet

1417-1419 conquête de la Normandie par Henri V

[avant 1419] Cliponville est un fief noble venant de la famille de Marie de Calleville, femme d'Hector de Flavy

[après 1419] Michel Dumont occupe le fief de Cliponville

21 mai 1420 traité de Troyes entre Charles VI et Henri V: instauration de la double monarchie

17 juillet 1429 Charles VII sacré à Reims

22 août 1429 Charles VII proclame l'édit de Compiègne sur le retour des biens à leurs anciens propriétaires

[après 1432, décès de Michel Dumont] Cliponville revient à Colin Dumont

8 mars 1439 accord entre les couples Robert Le Vigneron-Jehanne Dumont et Thomas Colombel-Maline Malleterre: Cliponville est désormais occupé par les Colombel

1449-1450 reconquête de la Normandie par Charles VII

[1449/1450] réinstallation d'Hector de Flavy et Marie de Calleville; clameur de haro prononcée par Thomas Colombel et Maline Malleterre

28 octobre 1450 confirmation de l'édit de Compiègne par Charles VII, enregistré en février 1451

[1450-1454] premier procès aux assises de Caux

30 décembre 1454 inversion de l'accord familial de 1439: Robert Le VigneronJehanne Dumont reprennent Cliponville

[1459-1461] second procès aux assises de Caux: jugement de Robert Hellart, lieutenant général du bailli de Caux, Guillaume Prunelay

23 avril 1464 Nicolas Lecomte, procureur d'Hector de Flavy, sous le sceau de la prévôté de Paris

20 mai 1464 accord entre les Flavy et les Choinet: Cliponville revient aux Choinet pour Thomas, le fils de Jehanne Dumont et Robert Le Vigneron; Pierre Choinet verse 100 écus d'or à Hector de Flavy

25 décembre 1464 date-butoir de remise par Flavy à Choinet de tous les papiers touchant Cliponville 
Annexe 4-Liste des noms et fonctions par ordre d'apparition dans l'acte passé devant les tabellions de Rouen le dimanche 20 mai 1464

\begin{tabular}{|c|c|c|}
\hline & $\begin{array}{l}\text { Titres, grades, } \\
\text { fonctions, attributs } \\
\text { d'honneur }\end{array}$ & $\begin{array}{l}\text { Situation familiale et } \\
\text { patrimoniale; actions en justice }\end{array}$ \\
\hline Hector de Flavy & «Chevalier» & $\begin{array}{l}\text { Mari de Marie de Calleville } \\
\text { depuis 1430, acte «au droit d'elle» } \\
\text { pour le fief de Cliponville; présente } \\
\text { une doléance devant l'Échiquier }\end{array}$ \\
\hline $\begin{array}{l}\text { Marie } \\
\text { de Calleville }\end{array}$ & «Dame» & Femme d'Hector de Flavy \\
\hline $\begin{array}{l}\text { Thomas } \\
\text { Colombel }\end{array}$ & & $\begin{array}{l}\text { Second mari de Maline Malleterre, } \\
\text { prononce la clameur de haro }\end{array}$ \\
\hline $\begin{array}{l}\text { Maline } \\
\text { [Malleterre] }\end{array}$ & «au droit d'elle» & $\begin{array}{l}\text { Veuve de Michel Dumont, } \\
\text { remariée à Thomas Colombel, } \\
\text { prononce la clameur de haro }\end{array}$ \\
\hline Michel Dumont & $\begin{array}{l}\text { [bourgeois, avocat, } \\
\text { lieutenant du vicomte } \\
\text { de Rouen] }\end{array}$ & $\begin{array}{l}\text { Premier mari de Maline } \\
\text { Malleterre, possesseur du fief } \\
\text { de Cliponville, père de Colin } \\
\text { et Jehanne }\end{array}$ \\
\hline $\begin{array}{l}\text { Guillebert } \\
\text { Couppequesne }\end{array}$ & Tabellion 1458-1466 & $\begin{array}{l}\text { Prononce la «clameur de haro» } \\
{[1449 / 1450]}\end{array}$ \\
\hline $\begin{array}{l}\text { Robin } \\
\text { Le Vigneron }\end{array}$ & $\begin{array}{l}\text { [bourgeois, avocat, } \\
\text { conseiller en cour laie] }\end{array}$ & $\begin{array}{l}\text { Premier mari de Jehanne Dumont, } \\
\text { acte «au droit d'elle» }\end{array}$ \\
\hline $\begin{array}{l}\text { Jehanne } \\
\text { Dumont }\end{array}$ & «au droit d'elle» & $\begin{array}{l}\text { Fille de Michel Dumont } \\
\text { et Maline Malleterre } \\
\text { Femme de Robin Le Vigneron, } \\
\text { puis de Pierre Choinet }\end{array}$ \\
\hline Colin Dumont & [avocat] & $\begin{array}{l}\text { Fils de Michel Dumont } \\
\text { et Maline Malleterre } \\
\text { [héritier du fief de Cliponville] }\end{array}$ \\
\hline Pierre Choinet & $\begin{array}{l}\text { Honorable, maître, } \\
\text { docteur en médecine } \\
\text { [bourgeois, médecin } \\
\text { et astrologue } \\
\text { de Louis XI; auteur du } \\
\text { Rosier des guerres et du } \\
\text { Livre des trois âges] }\end{array}$ & $\begin{array}{l}\text { Second mari de Jehanne Dumont, } \\
\text { acte au «droit d'elle», procureur } \\
\text { de sa femme, règle l'affaire par un } \\
\text { compromis }\end{array}$ \\
\hline
\end{tabular}




\begin{tabular}{|c|c|c|}
\hline $\begin{array}{l}\text { Robert/ Robinet } \\
\text { Hellart }\end{array}$ & $\begin{array}{l}\text { Lieutenant général } \\
\text { du bailli de Caux }\end{array}$ & $\begin{array}{l}\text { Prononce le jugement du second } \\
\text { procès [1459-1451] aux assises de } \\
\text { Caudebec }\end{array}$ \\
\hline Nicole Lecomte & «Messire» & $\begin{array}{l}\text { Procureur d'Hector de Flavy } \\
\text { et Marie de Calleville, } \\
\text { règle l'affaire par un compromis }\end{array}$ \\
\hline $\begin{array}{l}\text { [Guillaume } \\
\text { Prunelay] }\end{array}$ & [seigneur d'Herbault] & Bailli de Caux [1459-1461] \\
\hline Pierre Chouart & $\begin{array}{l}\text { Notaire du roi } \\
\text { en son Châtelet de Paris }\end{array}$ & $\begin{array}{l}\text { Valide la procuration } \\
\text { de Nicole Lecomte }\end{array}$ \\
\hline Antoine Goupil & $\begin{array}{l}\text { Notaire du roi } \\
\text { en son Châtelet de Paris }\end{array}$ & $\begin{array}{l}\text { Valide la procuration } \\
\text { de Nicole Lecomte }\end{array}$ \\
\hline $\begin{array}{l}\text { Robinet } \\
\text { Delamare }\end{array}$ & & Témoin de l'acte du 20 mai 1464 \\
\hline $\begin{array}{l}\text { Robinet } \\
\text { Le Caron }\end{array}$ & & Témoin de l'acte du 20 mai 1464 \\
\hline
\end{tabular}

